\title{
Universidades y comunicación. Papel de Twitter durante el inicio de la crisis sanitaria de la Covid-19
}

\section{Universities and communication: role of Twitter during the beginning of the Covid-19 health crisis}

\author{
Marta Ferrer-Serrano; María-Pilar Latorre-Martínez; Raquel Lozano-Blasco
}

Cómo citar este artículo:

Ferrer-Serrano, Marta; Latorre-Martínez, María-Pilar; Lozano-Blasco, Raquel (2020). “Universidades y comunicación. Papel de Twitter durante el inicio de la crisis sanitaria de la Covid-19". Profesional de la información, v. 29, n. 6, e290612.

https://doi.org/10.3145/epi.2020.nov.12

Artículo recibido el 07-05-2020

Aceptación definitiva: 09-06-2020

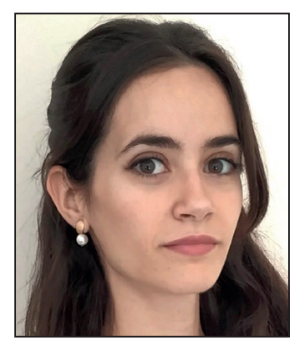

Marta Ferrer-Serrano $\square$

https://orcid.org/0000-0002-1859-0518

Universidad de Zaragoza

Facultad de Economía y Empresa

Gran Vía, 2

50005 Zaragoza, España

martafe@unizar.es

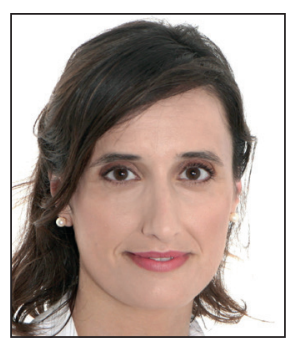

María-Pilar Latorre-Martínez https://orcid.org/0000-0002-8486-6885

Universidad de Zaragoza

Facultad de Ciencias Sociales y del Trabajo

Violante de Hungría, 23

50009 Zaragoza, España

latorrep@unizar.es

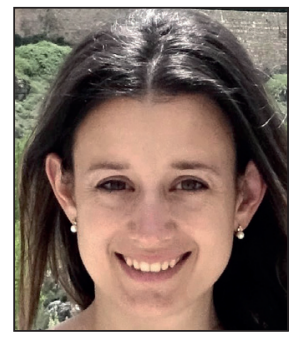

Raquel Lozano-Blasco

https://orcid.org/0000-0002-0100-1449

Universidad de Zaragoza

Facultad de Educación

Pedro Cerbuna, 12

50009 Zaragoza, España

rlozano@unizar.es

\section{Resumen}

La crisis derivada de la pandemia de la Covid-19 ha afectado, entre otros colectivos, a las universidades ya que han visto modificadas sus actividades habituales. Al mismo tiempo las redes sociales y en particular Twitter, han experimentado un aumento de su uso. Por ello, el objetivo de esta investigación consiste en analizar el comportamiento de las universidades españolas en Twitter entre el 1 de enero y 30 de abril de 2020. Se han monitorizado las cuentas corporativas de todas las universidades españolas, se han analizado los key performance indicators (KPIs) más relevantes (compromiso, seguidores, tweets, favoritos y retweets), y se ha analizado el sentimiento de los tweets con mayor impacto. Se propone el modelo "educativos, cooperativos, corporativos y divulgativos" (ECCD) capaz de categorizar el tipo de información emitido por las universidades en situaciones de crisis. Se constata la relevancia de Twitter como canal comunicativo en situaciones críticas, así como el aumento del compromiso de la comunidad digital con las cuentas universitarias. Se ha demostrado cómo la polaridad positiva de los mensajes emitidos por universidades de titularidad privada aumenta en situaciones de crisis. Por último, se han observado diferentes patrones comportamentales en la etapa anterior al Covid-19 y en la etapa de pandemia.

Financiación

Esta publicación ha sido cofinanciada por el Fondo Europeo de Desarrollo Regional a través del programa Programa Interreg V-A España-Francia-Andorra-Poctefa (proyecto Retsaso EFA341/19). El objetivo del Poctefa es reforzar la integración económica y social de la zona fronteriza España-Francia-Andorra. Su ayuda se concentra en la creación de una red transfronteriza de actores de la intervención sanitaria y social por medio de una plataforma colaborativa de trabajo y de una oferta transfronteriza de formación.
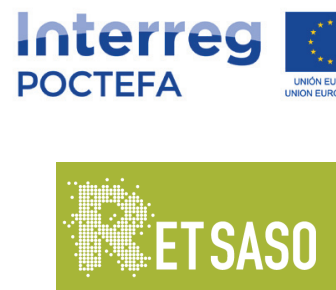


\title{
Palabras clave
}

Universidades; Covid-19; Coronavirus; Pandemias; Emergencias sanitarias; Epidemias; Crisis sanitarias; Alertas sanitarias; Redes sociales; Medios sociales; Comunicación; Confinamiento; Comunidades digitales; Modelo ECCD; Twitter; Fanpage Karma.

\begin{abstract}
The crisis resulting from the Covid-19 pandemic has affected (among other organizations) universities, because their usual activities have been modified. Social networks, in particular Twitter, have experienced an increase in use. The aim of this research is to analyze the behavior of Spanish universities on Twitter during the period between 1 January and 30 April 2020. The corporate accounts of all Spanish universities were monitored, the most relevant key performance indicators (KPIs: commitment, followers, tweets, favorites, and retweets) were analyzed, and finally, a sentiment analysis of the tweets with the greatest impact was carried out. In addition, this study proposes the ECCD model (educational, cooperative, corporate and informative), which is capable of categorizing the type of information issued by universities in crisis situations. This article shows the relevance of Twitter as a communication channel in critical situations, as well as the increased commitment of the digital community to university accounts. It also demonstrates how the positive polarity of messages issued by privately owned universities increases in crisis situations. Finally, this article highlights different behavior between the pre-Covid-19 compared with the pandemic stage.
\end{abstract}

\section{Keywords}

Universities; Covid-19; Coronavirus; Pandemics; Health emergencies; Epidemics; Health crisis; Health alerts; Social networks; Social media; Communication; Confinement; Digital communities; ECCD framework; Twitter; Fanpage Karma.

\section{Introducción}

La Organización Mundial de la Salud declaró la emergencia de salud pública de interés internacional debida a la Covid-19 en enero de 2020. El 14 de marzo, ante la velocidad de propagación, la alta dispersión geográfica y la gravedad de algunos casos informados, la OMS declaró la pandemia mundial. Como consecuencia, las naciones fueron tomando medidas de contención. En particular, España declaró el Estado de alarma a través de la publicación del Real decreto 463/2020, de 14 de marzo (España, 2020), que obligaba al confinamiento de la población.

Estas medidas han afectado a diferentes colectivos que se han visto en la obligación de reorganizar sus actividades habituales y adaptarlas virtualmente. Los centros de educación y en concreto, las universidades suspendieron las actividades docentes presenciales y las actividades de investigación no esenciales. El paso a la docencia online generó un alto nivel de nerviosismo añadido a la incertidumbre derivada del confinamiento a los colectivos involucrados (Hiremath et al., 2020).

La red social Twitter se ha erigido como un canal eficaz de transmisión rápida de información en momentos de crisis como pandemias, catástrofes naturales o crisis humanitarias (Fernández-Luque; Imran, 2018; Liu; Lai; Xu, 2018; Pourebrahim et al., 2019) que permite medir la sensibilidad de la población ante un suceso adverso (Theja-Bhavaraju; Beyney; Nicholson, 2019). Al igual que en crisis previas, esta situación ha ocasionado un interesante repunte del uso de medios comunicativos (Casero-Ripollés, 2020).

En este estudio se monitorizan las cuentas corporativas de todas las universidades españolas durante el período comprendido entre el 1 de enero y el 30 de abril de 2020. Este período permite analizar los comportamientos organizativos en la etapa anterior a la pandemia y en la etapa de confinamiento más severo, así como identificar el papel que ha jugado Twitter como canal comunicativo para las universidades. Además, dado que la bibliografía académica expone que pueden existir diferencias entre las organizaciones según su titularidad, privadas y públicas (Liu; Lai; Xu, 2018), se realiza dicha distinción. Por otra parte, se plantea el modelo ECCD (nombre que surge de las iniciales: educativos, cooperativos, corporativos y divulgativos) que permite categorizar la información de Twitter en función de la intencionalidad del mensaje.

Este artículo se articula en las siguientes secciones: elaboración del marco teórico y preguntas de investigación; explicación de la metodología; descripción y exposición de los resultados obtenidos; discusión de estos de acuerdo con la bibliografía previa, y conclusiones más relevantes derivadas del estudio.

\section{Marco teórico}

Algunas fuentes oficiales informan de que el primer caso conocido portador de coronavirus fue identificado en Wuhan, China, en noviembre de 2019 (NHPCO, 2020). A partir de ese momento la propagación del virus, en primera instancia local y posteriormente mundial, derivó en la adopción de medidas sanitarias de contención con el objetivo de evitar el colapso de los sistemas sanitarios. En España en particular, el día 14 de marzo del 2020, se publicó el Real decreto 463/2020 (España, 2020) que imponía el Estado de alarma junto con numerosas indicaciones de obligado cumplimiento,
Se plantea el modelo ECCD que permite categorizar la información de Twitter en función de la intencionalidad del mensaje: educativo, cooperativo, corporativo o divulgativo 
entre otras: confinamiento de la población, cierre de fronteras y suspensión del trabajo presencial y su adaptación al teletrabajo. La adecuación de la docencia presencial a la virtual (Hiremath et al., 2020) así como el apoyo social y emocional al estudiantado, han supuesto un reto para la comunidad educativa (Wang; Ng; Brook, 2020).

La bibliografía académica ha señalado que las redes sociales representan una nueva arquitectura en la que intervienen actores con nuevos roles sociales (Sedereviciute; Valentini, 2011; Trainor et al., 2014). A este respecto, la comunidad digital ha surgido como un actor clave (Churchill; Nelson; Denoue, 2003; Katz; González, 2016). Por otra parte, el uso de las redes sociales permite la generación de un gran volumen de datos que pueden analizarse y utilizarse para crear información valiosa para diferentes organizaciones (Wamba et al., 2015; Jin et al., 2015). Barret et al., (2015) hallaron que el creciente desdibujamiento de las fronteras entre las instituciones y las comunidades online puede crear oportunidades para que estas últimas desempeñen un papel más importante en la creación de valor. En los últimos años los investigadores han prestado mayor atención a la comprensión de las nuevas relaciones que han surgido como resultado de la aparición de las redes sociales, así como al impacto que los factores contextuales tienen en un ecosistema determinado (González-Bailón; Wang, 2016; Kim; Hastak, 2018).

Tradicionalmente, los estudios que analizan las redes sociales las han medido a través de indicadores clave de rendimiento, también conocidos como KPI (Keegan; Rowley, 2017). Los más utilizados son número de likes, retweets, engagement o compromiso, número de fans, lealtad y rendimiento (Latorre-Martínez; Orive-Serrano; Íñiguez-Dieste, 2018). Otro indicador que ha interesado a la comunidad científica y que aporta valiosa información cualitativa es el llamado "análisis de sentimiento" (Yu; Duan; Cao, 2013; Ceron et al., 2014). Resulta de interés el estudio de la polaridad de los mensajes que informa sobre comportamientos particulares y complejos. Además, de acuerdo con Thelwall y Levitt (2020), dar a conocer la polaridad de los mensajes transmitidos en Twitter puede ser importante para conseguir cambios de gestión en respuesta.

La pandemia de la Covid-19 ha supuesto la modificación de las relaciones de todos los grupos de edad: niños (Weaver; Wiener, 2020), ancianos (Mehra et al., 2020), jóvenes y adultos (Moghanibashi-Mansourieh, 2020; Wang; Tang, 2020). Los estudiantes universitarios son quienes mayores niveles de "infodemia" presentan (Gao et al., 2020); en otras palabras, reacción emocional adversa ante las continuas noticias de naturaleza catastrofista, concepto que ya fue defendido por Everts (2013) durante la pandemia por gripe porcina (2009-2010). La vida en confinamiento ha ocasionado el incremento exponencial del uso de las redes sociales como canales que cumplen funciones sociales básicas: relación y despedida (Wallace et al., 2020), información (NHPCO, 2020) y ocio.

La necesidad continua de actualización de información vertebra a las redes sociales como elementos clave en la gestión de crisis (Fernández-Luque; Imran, 2018; Liu; Lai; Xu, 2018; Pourebrahim et al., 2019), viendose incrementada la actividad en las cuentas corporativas de instituciones gubernamentales durante catástrofes naturales o crisis humanitarias, y reduciéndose una vez conclusa (Pourebrahim et al., 2019). Del mismo modo, Twitter ha permitido ante situaciones semejantes monitorizar y comprender los sentimientos de la sociedad (Takahashi; Tandoc; Carmichael, 2015; Vijaykumar et al., 2018). Ejemplo de ello fue el huracán Sandy: Pourebrahim et al., (2019) encontraron cómo los sentimientos positivos aparecieron antes y después del suceso, mientras que durante el pico prevalecieron los negativos. Por otra parte, Singh, Roy y Gangopadhyay (2019) exponen cómo los sentimientos y emociones expresados en Twitter durante el tiroteo en Las Vegas en 2017 evolucionaron con el tiempo, de manera que el enfado, el disgusto y la tristeza crecieron rápidamente para reducirse progresivamente.

Los incipientes datos sobre el papel de las redes sociales en la pandemia de la Covid-19 explican cómo surgen nuevas funciones en este tipo de plataformas, mientras que otras se intensifican (Thelwall; Levitt, 2020). Wallace et al. (2020) explican que las redes sociales son en frecuentes ocasiones la única vía de despedirse de un ser querido y por tanto tienen un papel clave en el proceso de luto. Roy et al. (2020) exponen que sus participantes tenían un nivel moderado de concienciación sobre los síntomas y propagación de la Covid-19, así como una conciencia adecuada sobre su prevención. Sugieren que estos datos se deben al énfasis de medios de comunicación y gobierno en exponer sus medidas. Por otra parte, Barkur, Vibha y Kamath (2020) realizaron un estudio sobre los sentimientos de los conciudadanos en la India a partir de Twitter, encontrando que el sentimiento mayoritario durante la pandemia fue el optimismo, seguido de la confianza en su estado.

La aplicabilidad de las redes sociales en gestión de crisis puede ser trasladada a la comunidad educativa, ya que estas tienen un potencial como elemento de transmisión de información académica (Forkosh-Baruch; Hershkovitz, 2012). En particular, Dabner (2012) defiende en su investigación cómo las redes sociales fueron los canales determinantes tras el terremoto de 7,1 de magnitud que asoló Nueva Zelanda. Durante meses fueron la vía de coordinación y comunicación de la University of Canterbury (Dabner, 2012). La respuesta fue a la par rápida y eficaz, permitiendo la transmisión de información en una situación de catástrofe natural. Los estudiantes tuvieron una respuesta comprometida, si bien el autor señala la necesidad de mejorar la coordinación entre los encargados de tomar decisiones (Dabner, 2012). Del mismo modo Oradini y Saunders (2008) refuerzan la necesidad de que la red social sea abierta e interactiva, apostando también por la divulgación de conocimiento científico (López-Pérez; Olvera-Lobo, 2016). Rodríguez-Vázquez, Teijeiro-Álvarez y Blázquez-Lozano (2018) exponen como estas plataformas se han profesionalizado en las universidades españolas, prioritariamente en las de titularidad pública. Por otra parte, aunque el principal factor de motivación de redes sociales es 
su comunicación rápida y eficaz, la preocupación por la pérdida de privacidad es el elemento principal que inhibe este comportamiento en profesores (Akçayır, 2017). Por otra parte, es necesario remarcar el carácter humanitario de estas organizaciones, resaltando el ensalzamiento de conductas prosociales o apoyo a las víctimas (Coombs, 2007; Coombs et al., 2010), favoreciendo la estructuración de lazos comunitarios (Saxton; Guo, 2014).

El incremento experimentado por parte de la comunidad educativa no debe obviar la naturaleza especial de su alumnado pues son nativos digitales (White; Le-Cornu, 2011). Esto significa que el uso de redes sociales forma parte de su cotidianidad, al punto de ser intrínsecamente necesarias para comprender su generación (Grimal, 2020), llegando a padecer síntomas específicos como "fear of missing out" (Hetz; Dawson; Cullen, 2015), nomophobia (King et al., 2013), o estrés (Qiu et al., 2020), todos ellos relacionados con la adicción a estas plataformas (Cheng; Li, 2014). A esta situación se añade la relevancia de la edad como elemento determinante en el malestar e inestabilidad psico-social derivada de la Covid-19, siendo los adultos jóvenes el grupo más vulnerable (Ahmed et al., 2020; Moghanibashi-Mansourieh, 2020). En coherencia, las universidades han apostado por realizar no sólo un cambio metodológico en su docencia sino también ejecutar un acompañamiento emocional a sus estudiantes (Wang; Ng; Brook, 2020).

La situación de excepcionalidad que está viviendo la comunidad educativa exige analizar los distintos comportamientos organizativos, así como identificar el papel que ha jugado Twitter como canal comunicativo. Además, resulta relevante estudiar con más detenimiento los sentimientos detrás de los mensajes más valorados. Por ello se considera necesario analizar de manera cualitativa el significado emocional de los tweets más compartidos, atendiendo a los criterios de Kireyev, Palen y Anderson (2009) sobre los hashtags y palabras clave en la fase anterior a la epidemia y durante esta. De esta forma, según el léxico empleado proponemos un modelo teórico que se articula en los siguientes principios:

- Twitter es un canal eficaz de transmisión rápida de información en momentos de crisis (Fernández-Luque; Imran, 2018; Liu; Lai; Xu, 2018; Pourebrahim et al., 2019) que permite medir la sensibilidad de la población ante dicho suceso (Theja-Bhavaraju; Beyney; Nicholson, 2019).

- Los mensajes generados en redes sociales durante catástrofes naturales o crisis humanitarias se fundamentan en la transmisión de información y emociones surgidas como consecuencia de la situación vivida (Kireyev; Palen; Anderson, 2009).

- El ser humano ante situaciones difíciles adquiere un comportamiento prosocial de apoyo a la comunidad (Glassman, 2000).

- Las organizaciones no están exentas y participan activamente, si bien las organizaciones que dependen del Estado centrarán sus esfuerzos en mostrar transparencia y mensajes cargados de compasión hacia las víctimas mientras que las organizaciones privadas tratarán de mantener en la mayor medida su imagen corporativa (Liu; Lai; Xu, 2018).

- Las universidades son organizaciones con unas características propias independientes de su titularidad, siendo la innovación, la ciencia, la investigación, la tolerancia y la integridad aspectos que definen tanto su ser como sus actuaciones.

Con esto en mente se propone el modelo ECCD, que garantiza los principios de fiabilidad específicos de Kappa (Fleiss; Cohen, 1973) entre las investigadoras, alcanzando niveles de acuerdo del 95\%. Este modelo pretende estudiar la respuesta de las universidades frente a una situación novedosa, en nuestro caso, durante la crisis sanitaria. El modelo ECCD permite la categorización de los mensajes emitidos en cuatro temas de acuerdo con su significado e intencionalidad, a las palabras y a los hashtags más empleados:

- Educativos: transmisión de información que afecta directamente al alumnado.

- Cooperativos: expresión de sentimientos de colaboración, agradecimiento, altruismo, cooperación y compasión hacia la ciudadanía.

- Corporativos: transmisión de información corporativa y neutra con el objetivo de mostrar el papel activo e imagen de las universidades.

- Divulgativos: comunicación de información con escaso valor informativo pero que pretende mantener el compromiso con la comunidad digital.

En consecuencia, el objetivo general de este artículo es la identificación de la posición en las redes sociales de las universidades españolas en las etapas iniciales de la pandemia. A su vez, el estudio propone las siguientes preguntas de investigación: ¿Qué papel ha ejercicio Twitter como red social principal de las universidades españolas durante la pandemia? ¿Existe un incremento claro del uso de Twitter por parte de las universidades durante el confinamiento? ¿Los mensajes lanzados presentan una polaridad positiva o negativa? ¿Existen diferencias entre las dos etapas objeto de análisis? ¿Existen comportamientos diferentes en función de la titularidad de las universidades? De acuerdo con el modelo ECCD, ¿Qué tipo de intencionalidad se percibe en los mensajes emitidos en las distintas etapas? ¿Existen diferencias entre universidades privadas y públicas?

Estas preguntas de investigación plantean las siguientes hipótesis:

H1. La crisis sanitaria de la Covid-19 ha generado un aumento del uso de Twitter por parte de las universidades españolas.

H2. Los mensajes lanzados presentarán una polaridad positiva que se verá intensificada en la segunda etapa analizada.

H3. Los mensajes emitidos durante la pandemia serán en su mayoría de corte cooperativo.

H4. La titularidad de las universidades permitirá evidenciar diferencias significativas en los resultados. 


\section{Metodología}

Este estudio se ha ejecutado en tres fases (figura 1): recopilación de datos, análisis e interpretación de resultados. Se han monitorizado los perfiles corporativos de todas las universidades españolas (Anexo 1 ) en el período comprendido entre el 1 de enero y 30 de abril de 2020, posibilitando observar el comportamiento de estas instituciones antes y durante la crisis sanitaria de la Covid-19. El análisis se ha realizado mediante la aplicación Fanpage Karma, que posibilita la captación y monitorización de posts y tweets a la par que permite su análisis.

Los KPIs (indicadores clave de rendimiento o medidores de desempeño) analizados en este artículo, resultan de especial relevancia para el análisis de las interacciones en las redes sociales (Latorre-Martínez; Orive-Serrano; Íñiguez-Dieste, 2018). Son los siguientes:

- compromiso: que se calcula dividiendo la cantidad diaria de retweets y favoritos por el número de seguidores;

- número de seguidores;

- total de publicaciones;

- total de favoritos;

- total de retweets.

Posteriormente se ha utilizado la aplicación Meaning cloud y el pack Emotion recognition para analizar el tono emocional expresado en los mensajes emitidos con mayor impacto. De esta forma se estudia la polaridad, es decir, el software estudia las palabras del tweet y las clasifica según su positividad o negatividad (Hu; Liu, 2004). Las redes sociales como Twitter rebasan la mera función comunicativa y trascienden a un plano más emocional, donde las personas no sólo comunican y transmiten información actual, sino que expresan opiniones y sentimientos. Es por ello esencial estudiar el indicador de análisis de sentimiento para comprender de manera holística la visión de una población (Yu; Duan; Cao, 2013; Ceron et al., 2014) y concretamente durante estados de emergencia que permitan mejorar tanto la gestión de esta como entender las respuestas sociales de una comunidad (Ruz; Henríquez; Mascareño, 2020). Finalmente se propone el modelo ECCD y categorizan los tweets de mayor impacto durante las dos etapas permitiéndonos extraer interesantes conclusiones.

\section{Resultados}

\subsection{Análisis de KPIs y descriptivo}

En el Anexo 2 se recogen los resultados obtenidos del análisis de los KPIs 1-5 para cada universidad española durante el período analizado. En términos generales, los niveles de compromiso de la comunidad digital con las universidades son bajos y presentan cierta variabilidad si atendemos a la desviación típica. Este indicador evidencia que, al margen del tipo de comunicación difundida por las universidades y su utilidad en la situación de crisis, existe una escasa interacción de los seguidores con las cuentas universitarias. En concreto, son cuatro las organizaciones que destacan con porcentajes superiores al 0,40 por el compromiso que tienen con su comunidad digital: las universidades Politécnica de Cartagena, Zaragoza, Jaume I y Oviedo. En cambio, observamos cómo no hay una correlación lineal entre compromiso y número de seguidores. Las universidades que encabezan el ranking de número de fans son las de Granada, Sevilla, Málaga, UNED, Murcia y Alicante. Todas superan los 100.000 seguidores en Twitter. Si prestamos atención a las interacciones recibidas en términos de "favoritos" y de "retweets", las universidades de Sevilla, Salamanca, Complutense de Madrid, UNED, Autònoma de Barcelona y Politécnica de Madrid destacan por encima del resto. Por último, cuando identificamos las

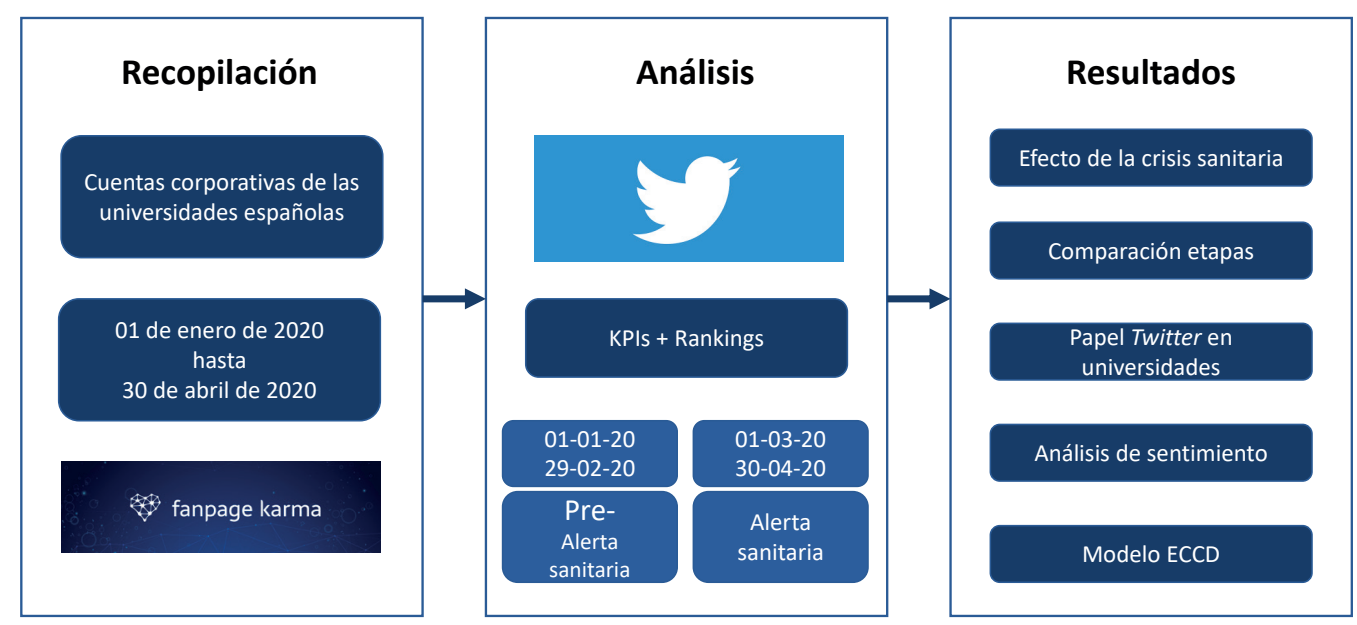

Figura 1. Diseño del estudio 


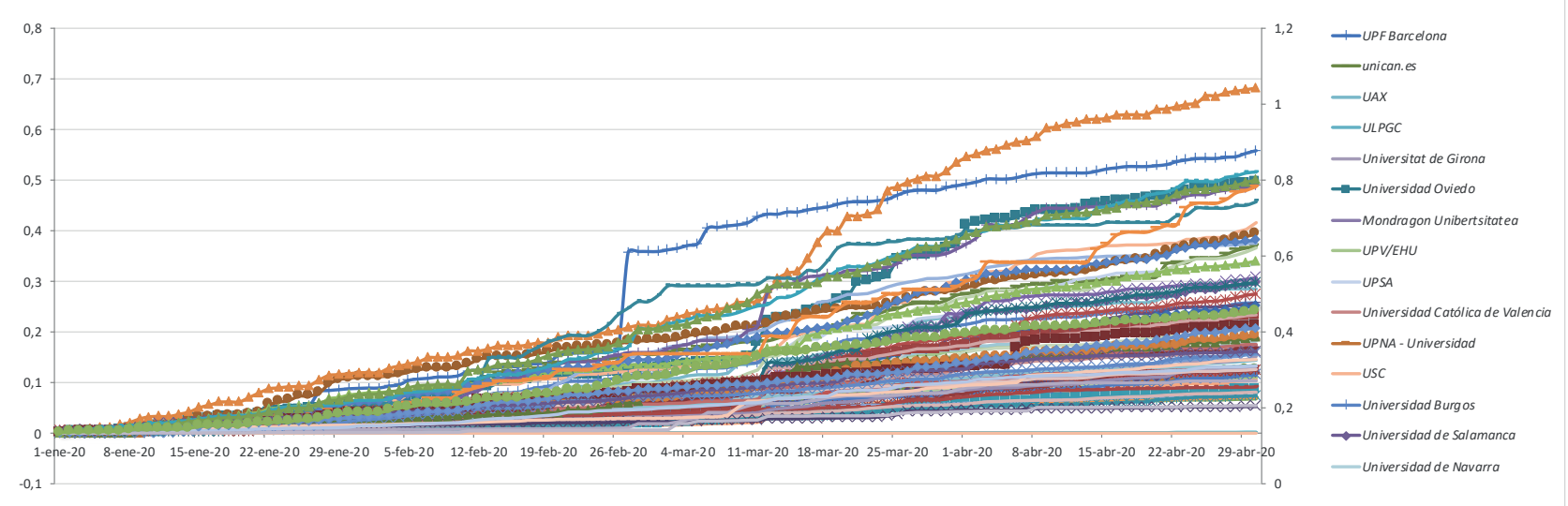

Figura 2. Compromiso de las universidades españolas en Twitter (enero-abril 2020)

universidades más activas en Twitter durante el período objeto de análisis, la Universidad de Sevilla (4.272 publicaciones) vuelve a posicionarse en primer lugar, la Universidad de Burgos (2.502 publicaciones) aparece en segundo puesto a pesar de no haber salido anteriormente, la Jaume I (2.475 publicaciones) en tercer lugar, y la de Murcia (2.100 publicaciones) en cuarta posición.

Resulta de especial relevancia analizar la tendencia de uso de Twitter durante el período objeto de análisis. La figura 2 muestra el compromiso de los seguidores en esta red social. La figura 3 muestra el crecimiento del número de fans a lo largo del período para cada perfil corporativo de las universidades españolas en Twitter. Como se puede apreciar durante el período estudiado han crecido tanto el número de seguidores como el compromiso tal y como la bibliografía académica previa parecía apuntar, permitiéndonos apoyar la Hipótesis 1.

Además, se han analizado los quince tweets que más "favoritos" han recibido y la cuenta de universidad a la cual se refieren (Anexo 3). Nueve tweets de los quince guardan relación con la pandemia. En particular, cuatro mencionan la suspensión de clases presenciales. Tres tweets sobre actividades de investigación para lograr reducir el contagio del coronavirus, uno sobre donaciones por parte de personajes públicos y uno alentando enviar material sanitario. Las universidades que han tenido mayor éxito a la hora de compartir sus tweets han sido las catalanas Pompeu Fabra, de Barcelona y Autònoma de Barcelona, siendo la comunidad universitaria catalana muy activa. Igualmente destaca la Universidad de Málaga, siendo una de más retweeteadas.

La tabla 1 resume los 5 posts con sólo texto, 5 posts con imagen y 5 posts con enlaces con mayor impacto en Twitter durante enero y febrero, que se correspondería con la etapa anterior a la pandemia y por otro lado marzo y abril, que correspondería con la fase de alerta sanitaria. Por un lado, en la segunda etapa analizada, se observa un claro aumento en el número de interacciones en la red social. En las tres categorías contemplamos una mayor actividad por parte de la comunidad digital. Por otro lado, la temática de los tweets también ha cambiado significativamente. En la primera etapa, distintos temas conviven mientras que en la segunda se podría hablar de un único tema relativo a la alerta sanitaria. Durante la alerta sanitaria las universidades han transmitido dos tipos de comunicados principalmente:

- relacionados con la docencia online;

- acciones prosociales como ceder medios materiales a sanitarios, generar respiradores o dar recomendaciones a la población, es decir, refuerzan públicamente acciones de cooperación y solidaridad.

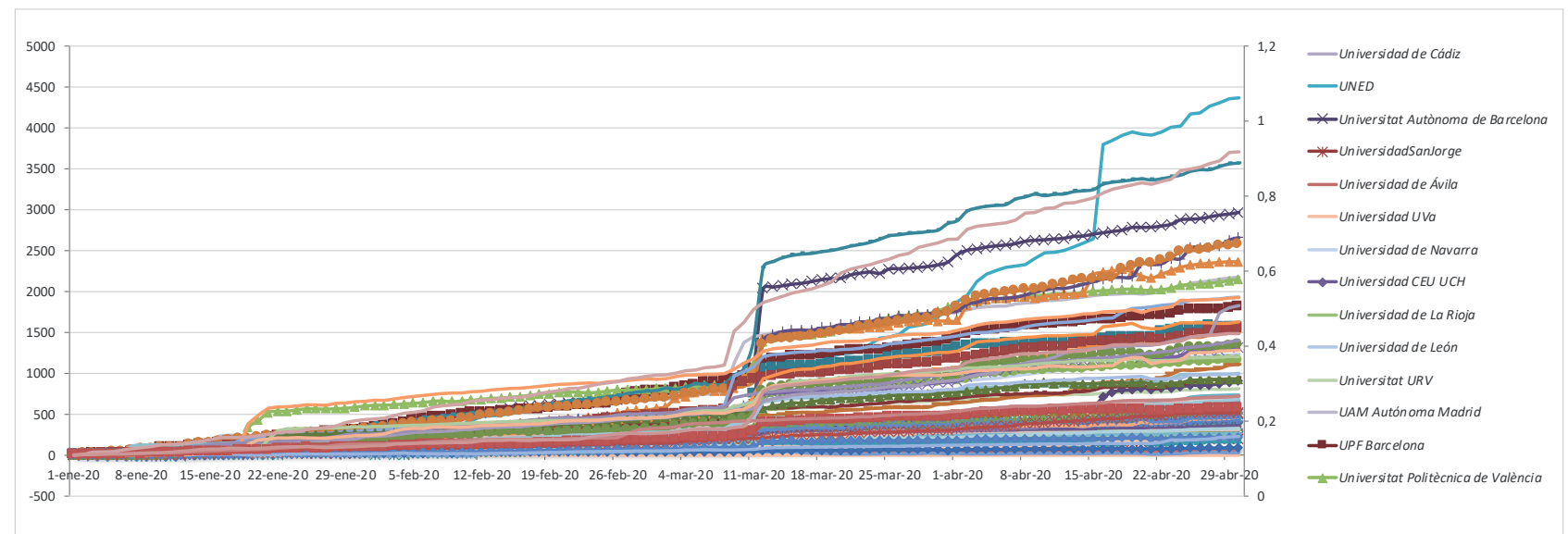

Figura 3. Crecimiento acumulado del número de seguidores de las universidades españolas en Twitter (enero-abril 2020) 
Tabla 1. Mensajes emitidos con mayor impacto en Twitter en enero y febrero frente a marzo y abril de 2020

\begin{tabular}{|c|c|c|c|}
\hline Textos & Imágenes & Enlaces & \\
\hline \multicolumn{4}{|c|}{ 01-01-2020 hasta 29-02-2020 } \\
\hline TOP TEXTOS & TOP IMÁGENES & \multicolumn{2}{|l|}{ TOP ENLACES } \\
\hline 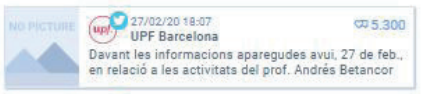 & 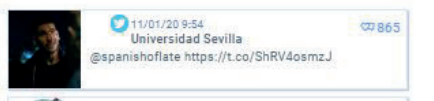 & $\begin{array}{l}\text { MTG - Music Technology } \\
\text { Group (UPF) } \\
\text { bit.ly/2RRFMco }\end{array}$ & 852 \\
\hline 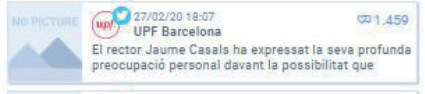 & 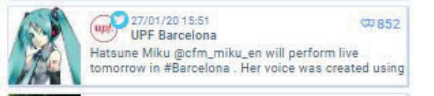 & $\begin{array}{l}\text { La UOC rebrà un } \\
\text { reconeixement especial en la } \\
\text { ow.ly/niqn50yka7K }\end{array}$ & 410 \\
\hline 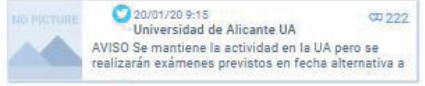 & 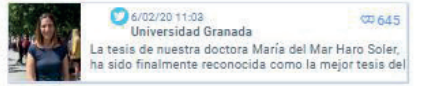 & $\begin{array}{l}\text { ShortURL-URL Shortener } \\
\text { shorturl.at/owCX4 }\end{array}$ & 314 \\
\hline 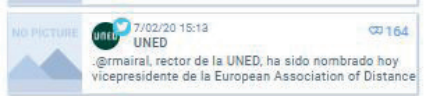 & 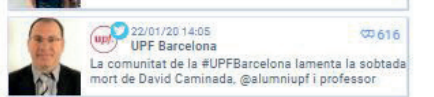 & $\begin{array}{l}\text { La Universidad de Alicante } \\
\text { conmemora el Día } \\
\text { s.ua.es/AXqO }\end{array}$ & $31:$ \\
\hline 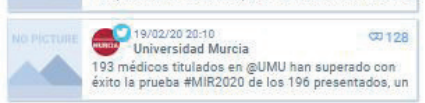 & 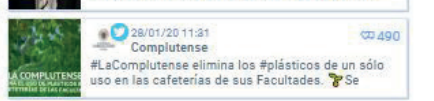 & $\begin{array}{l}\text { El premio Nobel Albert Fert se } \\
\text { incorpora a la Universidad del } \\
\text { bit.ly/20hleru }\end{array}$ & 253 \\
\hline & $01-03-2020$ hasta $30-04-2020$ & & \\
\hline TOP TEXTOS & TOP IMÁGENES & TOP ENLACES & \\
\hline 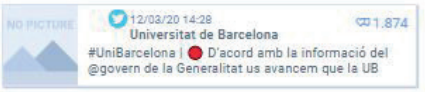 & 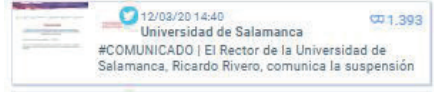 & $\begin{array}{l}\text { Antonio Banderas y Pablo } \\
\text { Alborán apoyan al grupo de la } \\
\text { uma.es/sala-de-prensa/noticias/ant.. }\end{array}$ & 1. \\
\hline 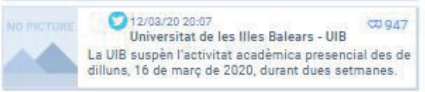 & 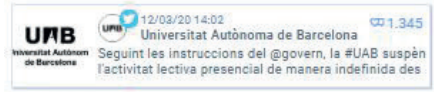 & $\begin{array}{l}\text { El prototipo 'Málaga Respira' } \\
\text { concluye con éxito el primer } \\
\text { uma.es/sala-de-prensa/noticias/el-.. }\end{array}$ & 1. \\
\hline 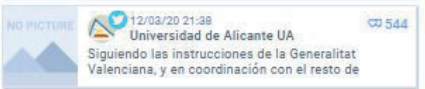 & 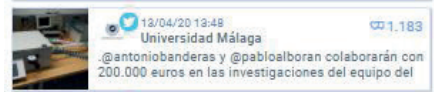 & $\begin{array}{l}\text { Angela Y. Davis, activista } \\
\text { politica nord-americana, serà } \\
\text { bittly/2ViPbqez }\end{array}$ & 971 \\
\hline 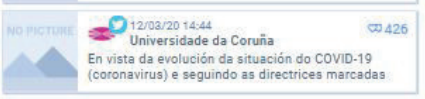 & 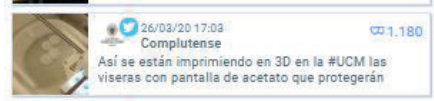 & $\begin{array}{l}\text { El respirador artificial } \\
\text { desarrollado por investigadores } \\
\text { uma.es/sala-de-prensa/noticias/el-r. }\end{array}$ & 957 \\
\hline 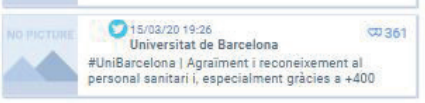 & 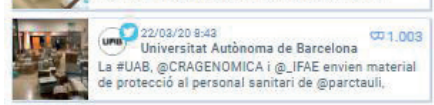 & $\begin{array}{l}\text { UNED UNED I COMUNICACIÓN } \\
\text { COMUNICADO COVID-19 } \\
\text { direc.to/dzq7 }\end{array}$ & 904 \\
\hline
\end{tabular}

Las palabras y hashtags utilizados con mayor frecuencia en el período anterior a la alerta sanitaria son multidisciplinares. Encontramos expresiones como "ciencia", "investigación”, "\#nosoloingenieria” o “\#diamujeryniñaenciencia” entre otros. En cambio, cuando analizamos el período de la pandemia, las palabras utilizadas se reducen prácticamente a un solo tema, la Covid-19 y la respuesta de las universidades a este. Algunos ejemplos son construcciones originadas en el seno de la pandemia y que constituyen lemas "\#YoMeQuedoEnCasa", "\#StopCovid-19" o "\#EsteVirusLoParamosUnidos" (tabla 2) empleados por los usuarios para exponer su compromiso con las medidas acordadas.

\subsection{Análisis de sentimiento}

Las figuras 4 y 5 representan los sentimientos según el nivel de positividad frente a negatividad (Hu; Liu, 2004). Se analizan los 100 mensajes de mayor impacto durante los dos primeros meses del año. Como se puede observar, la positividad

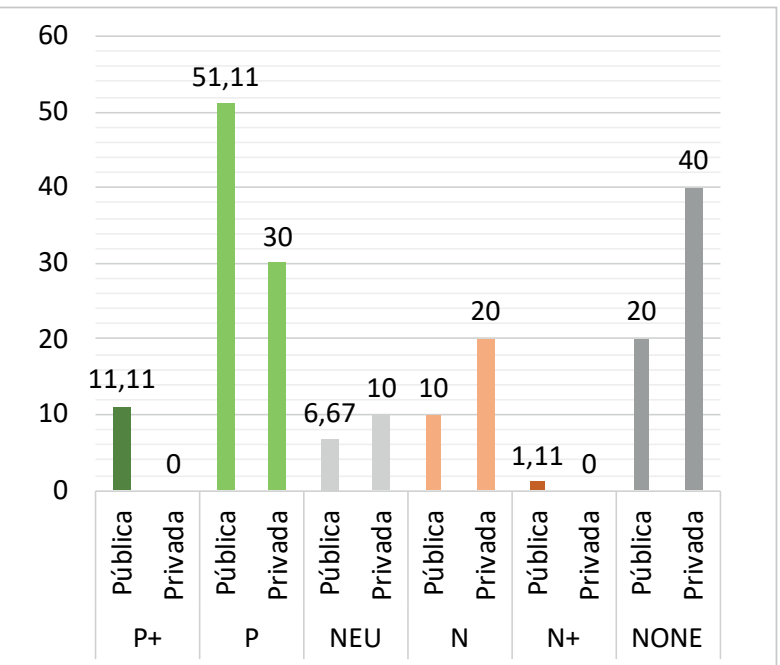

Figura 4. Polaridad diferenciada entre universidades públicas y privadas en enero y febrero.

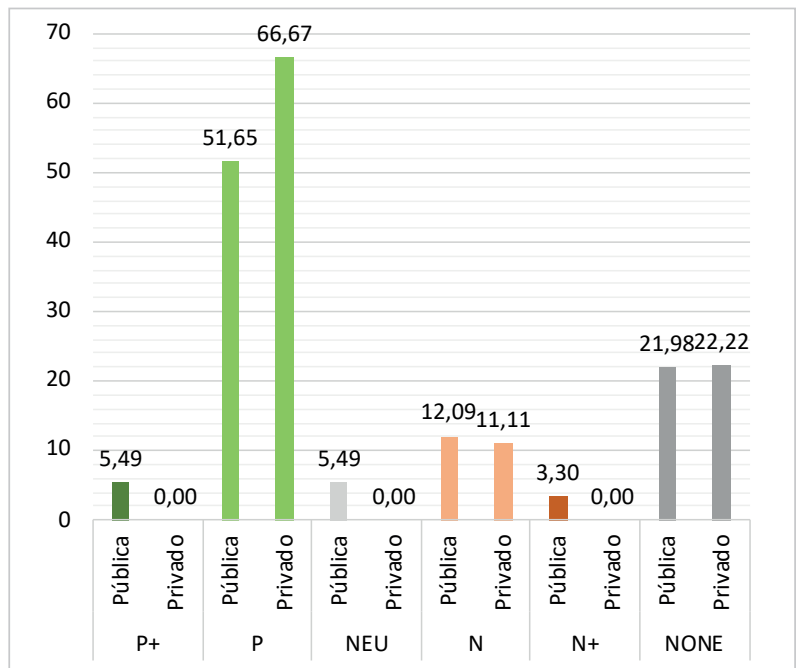

Figura 5. Polaridad diferenciada entre universidades públicas y privadas en marzo y abril. 
Tabla 2. Nubes de palabras más utilizadas en Twitter en enero y febrero frente a marzo y abril del 2020

\begin{tabular}{|c|c|}
\hline Hashtags más frecuentes & Palabras más utilizadas \\
\hline \multicolumn{2}{|c|}{ 01-01-2020 hasta 29-02-2020 } \\
\hline $\begin{array}{l}\text { HASHTAGS MÁS FRECUENTES } \\
\text { \#Unileon \#leonesp \#MeteoUBFisica } \\
\text { \#CICUS \#Cultura \#UBU \#Unioví \#Recerca } \\
\text { \#CampusGetafe \#URV \#LeamingFromFuture } \\
\text { \#universidad \#Noticias_UBU } \\
\text { \#ExpocienciaUnileon \#UAH \#Becas \#UPO } \\
\text { \#11F \#Mảlaga \#ODS } \\
\text { \#diamujeryniñaenciencia \#UdiMA \#Ciencia } \\
\text { \#deporte \#UAM \#UAB \#investigación } \\
\text { \#prácticas \#Grado \#SomUJI \#exposición } \\
\text { \#MUnibertsitatea \#mujeryciencia \#somuJi29 } \\
\text { \#Womeninscience \#UPFBarcelona \#Erasmus } \\
\text { \#Formación \#11F2020 \#Educaciön } \\
\text { \#LearningFromReality \#Estudiantes } \\
\text { \#ESPortada \#ErasmusPlus \#ucavila }\end{array}$ & $\begin{array}{l}\text { PALABRAS MÁS UTILIZADAS } \\
\text { María Hoy Congreso Sociales Santo Doctor UMU Grados } \\
\text { Comienza Comunidad Salas Gracias Nuevo Curso Máster } \\
\text { Aula Estudiantes Educación Garcia Ingenieria Ciencia } \\
\text { Jornadas Día Campus RT ABIERTO Tomás Juan } \\
\text { Instituto II Conferencia Española ULPGC Premios } \\
\text { Investigador Universidad Derecho UCAM Nuestro } \\
\text { Murcia Rectorado Universitaria Programa Universitat } \\
\text { España Internacional Escuela Centros Facultades } \\
\qquad \text { Mañana José Antonio } \\
\text { tamaño = frecuencia } \\
\text { verde }=\text { muchas reacciones } \\
\text { rojo }=\text { pocas reacciones }\end{array}$ \\
\hline \multicolumn{2}{|c|}{ 01-03-2020 hasta 30-04-2020 } \\
\hline 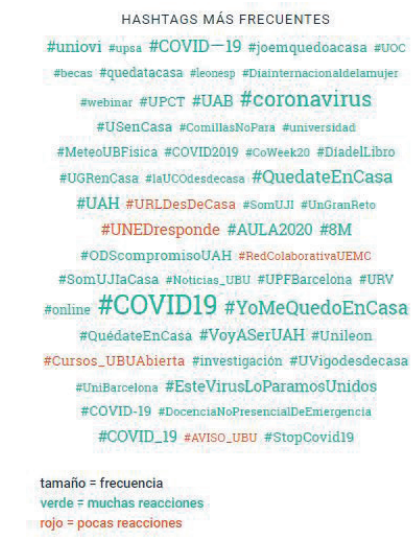 & $\begin{array}{l}\text { PALABRAS MÁS UTILIZADAS } \\
\text { José Vicerrectorado Aules Salud Rector Investigadores } \\
\text { Estudiantes Facultat Dia Educación Nuestro Social Nuevo } \\
\text { Gobierno Bibliotecas Servicio Online Internacional Avui } \\
\text { Püblicas Hoy Información COVID-19 Comunidad consulta } \\
\text { ULPGC Abierto RT Jomada Máster Facultad Santa } \\
\text { Centres Universitat Program Maria Buenos Hola } \\
\text { Universidad Universitaria Consejeria España Salut } \\
\text { Virtual Ciencia Campus Deporte Grado GRACIAS UCAM } \\
\text { Mañana Plan } \\
\text { tamaño = frecuencia } \\
\text { verde }=\text { muchas reacciones } \\
\text { rojo }=\text { pocas reacciones }\end{array}$ \\
\hline
\end{tabular}

Tamaño = frecuencia; Verde = muchas reacciones; Rojo = pocas reacciones

aumenta, especialmente en las universidades privadas a la par que la negatividad de sus tweets decrece, mientras que las universidades públicas no experimentan un crecimiento tan elevado y un leve repunte de negatividad. Es decir, la asimetría en polaridad aumenta durante el estado de alarma sanitario. Como consecuencia, se apoya la Hipótesis 2.

La casuística diferencial de esta situación exige el estudio en mayor profundidad de los tweets más compartidos mediante el modelo ECCD entre ambas franjas. Antes del inicio de la pandemia (figura 6) se observaba cómo independientemente de la titularidad, las universidades se centraban en transmitir información de tipo corporativo y divulgativo,

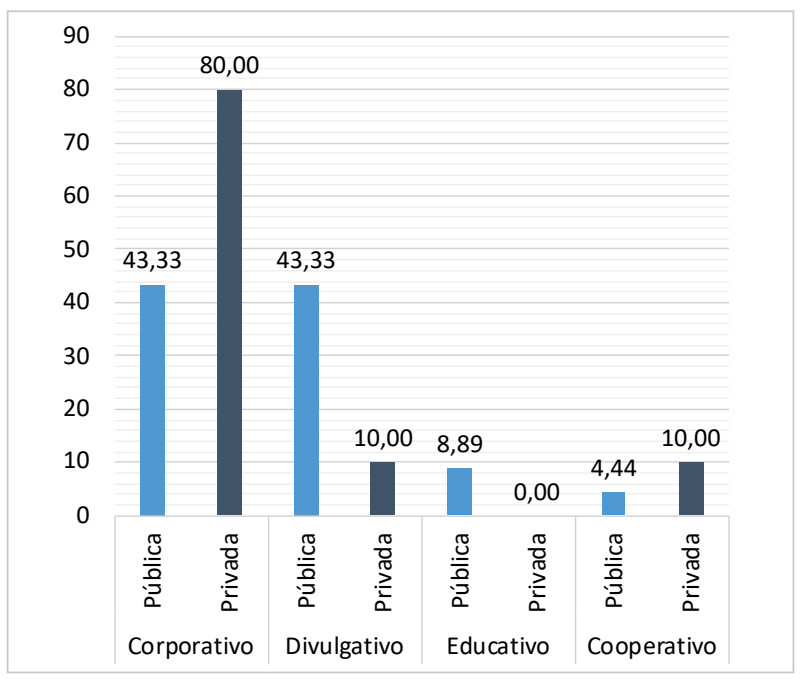

Figura 6. Categorización framework durante enero y febrero.

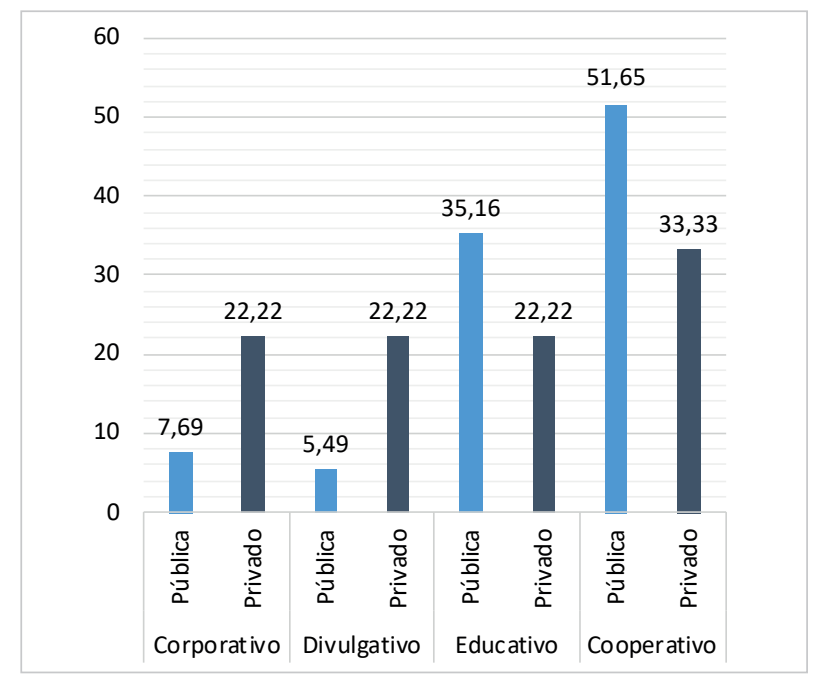

Figura 7. Categorización framework durante marzo y abril. 
mientras que las temáticas educativa y cooperativa eran claramente minoritarias. Sin embargo, la irrupción de la pandemia ha supuesto un notable cambio en los intereses de las universidades, invirtiendo la situación (figura 7). De esta forma, los tweets más compartidos hacen referencia a las modificaciones en la docencia y a la lucha cooperativa de la comunidad universitaria para frenar los estragos de la pandemia (se apoya la Hipótesis 3). Es decir, las universidades refuerzan públicamente actuaciones pro-sociales en las que participa su comunidad, bien estudiantes o profesores-investigadores en detrimento de información de tipo corporativo o divulgativo. De esta forma, Twitter se convierte en un espacio común para la comunidad universitaria donde expresar la esperanza y el ánimo para sobrellevar una situación catastrófica. La Hipótesis 4 es rechazada dado que a pesar de que se observan diferencias comportamentales en función de la titularidad de las universidades, no podemos decir que las diferencias sean los suficientemente significativas como para establecer un patrón claro.

\section{Discusión}

Las redes sociales son un canal digital de marcado carácter social. En particular, Twitter es más que una red de noticias breves que se comparten, es una plataforma que permite no sólo la expresión de un sentimiento u opinión, sino también compartir pensamientos, información objetiva y experiencias. De este modo, escuchar a redes sociales como Twitter permite comprender los sentimientos de una población (Ceron et al., 2014) e identificar las percepciones hacia un suceso o evento concreto (Ruz; Henríquez; Mascareño, 2020). Además, Twitter crea una comunidad digital cuyos lazos se estrechan mediante los retweets y likes (Saxton; Guo, 2014) a la vez que permite generar grupos en torno a una crisis (Aldoory; Grunig, 2012).

Si bien se ha mostrado el importante papel que juegan las instituciones como las universidades en redes sociales durante estas crisis, este fenómeno parece ser temporal. A este respecto, Pourebrahim et al., (2019) muestran cómo en una fase inicial en una situación crítica, el índice de usuarios creció, pero una vez concluido este período se perdió el compromiso con la población. Takahashi, Tandoc y Carmichael (2015) argumentan para el caso del tifón Haiyan que el compromiso en Twitter varía según el momento de su utilización y si el usuario está geográficamente cerca de la zona afectada (Theja-Bhavaraju; Beyney; Nicholson, 2019). Además, las pruebas indican que los usuarios de los medios de comunicación social valoran las fuentes tradicionales de los medios de comunicación en tiempos de crisis, dada la preponderancia de los tweets que hacen referencia a las fuentes de noticias de segunda mano y las vinculan con ellas.

Por otra parte, durante situaciones de emergencia el ser humano recupera su rol más social, ejecutando acciones basadas en la compasión, colaboración y cooperación (Glassman, 2000) las cuales revierten en el bienestar psicológico de la comunidad (Welton-Mitchell et al., 2018). Twitter se ha instaurado como un canal proclive para compartir experiencias, ideas y expresar sentimientos generados durante desastres naturales o crisis humanitarias (Fernández-Luque; Imran, 2018; Liu; Lai; Xu, 2018), de tal forma que la mayoría de los mensajes están marcados por información y las emociones despiertas durante la crisis (Kireyev; Palen; Anderson, 2009). En esta investigación podemos ver cómo el eje temático de las universidades cambia radicalmente antes y después del inicio de la crisis sanitaria, de manera que los tweets más compartidos se centran en la lucha común contra la Covid-19. Estas conductas refuerzan los lazos entre la comunidad, pues como explicamos el compromiso crece en todas las universidades españolas, reforzando el concepto de comunidad educativa (Aldoory; Grunig, 2012; Saxton; Guo, 2014; Reynard; Shirgaokar, 2019).

Las organizaciones no son ajenas a la pandemia. Como hemos mostrado, las universidades españolas brindan y refuerzan comportamientos solidarios como la cesión de material sanitario o la generación de respiradores a la vez que transmiten mensajes de compasión y colaboración hacia las víctimas (Coombs, 2007; Coombs et al., 2010). Si bien, esta situación no resta para que expresen una imagen corporativa, exponiendo al resto de comunidades su papel activo en la crisis sanitaria ya que reconocen públicamente la labor de investigadores que han modificado sus agendas para buscar antivirales, generar recursos sanitarios como respiradores o mostrar agradecimiento a los estudiantes que de manera voluntaria apoyan a los servicios sanitarios. Igualmente, existe una notable diferencia en las nubes de palabras pre y post Covid-19, especialmente en los hashtags más empleados. Los resultados son semejantes al estudio de Pourebrahim et al. (2019) durante el huracán Sandy en EUA. Del mismo modo, durante el Estado de alarma por la covid-19 destacaron:

- términos con relación directa con el suceso "sandy", "hurricane" versus "Covid19";

- términos propios y lemas como "frankenstorm" versus "Quédateencasa";

- términos que expresan cooperación y compasión "safe", "power", “help", "will”, "people”, “love”, "thank" versus "social", "nuestro", "gracia", "comunidad" o "ciencia". 
Es decir, en momentos de crisis, los usuarios comparten lemas grupales o palabras que permiten expresar su desacuerdo ante una realidad (Sola-Morales; Rivera-Gallardo, 2015) favoreciendo la generación de lazos (Saxton; Guo, 2014) y expresando su esperanza de volver a la normalidad.
Las universidades y sus colectivos han mostrado un aumento sustancial del uso de Twitter durante la pandemia

Respecto a la polaridad de sentimientos encontramos datos similares a los de Barkur, Vibha y Kamath (2020) en la población india durante el confinamiento por la Covid-19. Los mensajes más compartidos vienen marcados por la positividad. Si bien es necesario tener en cuenta que tantos los tweetteros más activos como los tweets más extensos tienen mayor carga emocional durante los estados de crisis (Reynard; Shirgaokar, 2019). Theja-Bhavaraju, Beyney y Nicholson (2019) en su estudio de simetría de polaridad de sentimientos en diversos acontecimientos catastróficos exponen la heterogeneidad de sus resultados y subrayan que la polaridad puede depender de la cercanía al desastre acaecido, aunque existe una gran diversidad, estando de acuerdo con Wu y Cui (2018).

\section{Conclusiones}

Esta investigación permite extraer importantes conclusiones. Se ha demostrado la relevancia de redes sociales como Twitter en situaciones críticas como la derivada de esta emergencia sanitaria. Gracias a la capacidad de intercambio inmediato de información, Twitter destaca como canal comunicativo. Particularmente, las universidades y sus colectivos, siendo uno de los grandes afectados por las medidas tomadas para hacer frente a la rápida propagación del virus, han mostrado un aumento sustancial del uso de Twitter durante la pandemia. Además, el compromiso de la comunidad digital con estas ha incrementado significativamente en las últimas semanas.

Por otro lado, se ha visibilizado el contenido de los mensajes con mayor impacto social y se ha analizado su polaridad positiva o negativa. En este sentido, a pesar de que la polaridad positiva de los mensajes emitidos por universidades de titularidad privada aumenta en situaciones de crisis, la diferencia no es lo suficientemente alta como para afirmar que existe un patrón comportamental.

Finalmente, se ha propuesto un modelo novedoso, que toma por nombre ECCD (educativo, cooperativo, corporativo y divulgativo), capaz de categorizar la información transmitida por las universidades en situaciones complicadas. El análisis de este modelo nos ha permitido identificar un claro cambio de comportamiento en el mensaje transmitido por las universidades en la etapa de emergencia sanitaria. Se ha pasado de emitir mensajes en los cuales se destaca la imagen corporativa, a una necesidad por emitir mensajes con un perfil cooperativo y pro-social, tratando de transmitir serenidad, ánimo y esperanza para sobrellevar esta situación.

Próximas investigaciones pueden centrar la atención en el análisis comportamental de las universidades en redes sociales en la etapa posterior a la alerta sanitaria, ya que como se ha debatido en este trabajo, existen evidencias de que el comportamiento y la intencionalidad de los mensajes emitidos, cambiarán.
Se ha identificado un cambio de comportamiento en las universidades: de mensajes en los que se destaca la imagen corporativa, a mensajes con un claro perfil cooperativo y pro-social

\section{Referencias}

Ahmed, Zahir; Ahmed, Oli; Aibao, Zhou; Hanbin, Sang; Siyu, Liu; Ahmad, Akbaruddin (2020). "Epidemic of Covid-19 in China and associated psychological problems". Asian journal of psychiatry, v. 51, 102092.

https://doi.org/10.1016/j.ajp.2020.102092

Akçayır, Gke (2017). "Why do faculty members use or not use social networking sites for education?". Computers in human behavior, v. 71, pp. 378-385.

https://doi.org/10.1016/j.chb.2017.02.028

Aldoory, Linda; Grunig, James E. (2012). "The rise and fall of hot-issue publics: Relationships that develop from media coverage of events and crises". International journal of strategic communication, v. 6, n. 1, pp. 93-108. https://doi.org/10.1080/1553118X.2011.634866

Barkur, Gopalkrishna; Vibha; Kamath, Giridhar B. (2020). "Sentiment analysis of nationwide lockdown due to Covid 19 outbreak: Evidence from India”. Asian journal of psychiatry, v. 51, 102089.

https://doi.org/10.1016/j.ajp.2020.102089

Barrett, Michael; Davidson, Elisabeth; Prabhu, Jaideep; Vargo, Stephen L. (2015). "Service innovation in the digital age: Key contributions and future directions". MIS quarterly, v. 39, n. 1, pp. 135-154.

https://doi.org/10.25300/MISQ/2015/39:1.03

Casero-Ripollés, Andreu (2020). "Impact of Covid-19 on the media system. Communicative and democratic consequences of news consumption during the outbreak". El profesional de la información, v. 29, n. 2, e290223.

https://doi.org/10.3145/epi.2020.mar.23 
Ceron, Andrea; Curini, Luigi; lacus, Stefano M.; Porro, Giuseppe (2014). "Every tweet counts? How sentiment analysis of social media can improve our knowledge of citizens' political preferences with an application to Italy and France". New media and society, v. 16, n. 2, pp. 340-358.

https://doi.org/10.1177/1461444813480466

Cheng, Cecilia; Li, Angel-Yee-lam (2014). "Internet addiction prevalence and quality of (real) life: A meta-analysis of 31 nations across seven world regions". Cyberpsychology, behavior, and social networking, v. 17, n. 12, pp. 755-760. https://doi.org/10.1089/cyber.2014.0317

Churchill, Elizabeth F.; Nelson, Les; Denoue, Laurent (2003). "Multimedia fliers: Information sharing with digital community bulletin boards". In: Huysman, Marleen; Wenger, Etienne; Wulf, Wolker (eds.). Communities and technologies. Dordrecht: Springer, pp. 97-117. ISBN: 9789048164189

https://doi.org/10.1007/978-94-017-0115-0_6

Coombs, W. Timothy (2007). "Protecting organization reputations during a crisis: The development and application of situational crisis communication theory". Corporate reputation review, v. 10, n. 3, pp. 163-176.

https://doi.org/10.1057/palgrave.crr.1550049

Coombs, W. Timothy; Frandsen, Finn; Holladay, Sherry J.; Johansen, Winni (2010). "Why a concern for apologia and crisis communication?". Corporate communications: an international journal, v. 15 n. 4, pp. 337-349.

https://doi.org/10.1108/13563281011085466

Dabner, Nicki (2012). “'Breaking ground' in the use of social media: A case study of a university earthquake response to inform educational design with Facebook". Internet and higher education, v. 15, n. 1, pp. 69-78.

https://doi.org/10.1016/j.iheduc.2011.06.001

España (2020). "Real decreto 463/2020, de 24 de marzo, por el que se declara el estado de alarma para la gestión de la situación de crisis sanitaria ocasionada por el Covid-19". BOE, n. 67, 14 marzo.

https://www.boe.es/buscar/act.php?id=BOE-A-2020-3692

Everts, Jonathan (2013). "Announcing swine flu and the interpretation of pandemic anxiety". Antipode, v. 45, n. 4, pp. $809-825$. https://onlinelibrary.wiley.com/doi/full/10.1111/j.1467-8330.2012.01021.x

https://doi.org/10.1111/j.1467-8330.2012.01021.x

Fernández-Luque, Luis; Imran, Muhammad (2018). "Humanitarian health computing using artificial intelligence and social media: A narrative literature review". International journal of medical informatics, v. 114, pp. 136-142.

https://doi.org/10.1016/j.ijmedinf.2018.01.015

Fleiss, Joseph L.; Cohen, Jacob (1973). "The equivalence of weighted Kappa and the intraclass correlation coefficient as measures of reliability". Educational and psychological measurement, v. 33, pp. 613-619. https://doi.org/10.1177/001316447303300309

Forkosh-Baruch, Alona; Hershkovitz, Arnon (2012). "A case study of Israeli higher-education institutes sharing scholarly information with the community via social networks". Internet and higher education, v. 15, n. 1, pp. 58-68. https://doi.org/10.1016/j.iheduc.2011.08.003

Gao, Junling; Zheng, Pinpin; Jia, Yingnan; Chen, Hao; Mao, Yimeng; Chen, Suhong; Wang, Yi; Fu, Hua; Dai, Junming (2020). "Mental health problems and social media exposure during Covid-19 outbreak". Plos one, v. 15, n. 4, pp. 1-10. https://doi.org/10.1371/journal.pone.0231924

Glassman, Michael (2000). "Mutual aid theory and human development: Sociability as primary". Journal for the theory of social behaviour, v. 30, n. 4, pp. 391-412. https://doi.org/10.1111/1468-5914.00137

González-Bailón, Sandra; Wang, Ning (2016). "Networked discontent: The anatomy of protest campaigns in social media". Social networks, v. 44, pp. 95-104. https://doi.org/10.1016/j.socnet.2015.07.003

Grimal, Richard (2020). "Are French millennials less car-oriented? Literature review and empirical findings". Transportation research part D: Transport and environment, v. 79.

https://doi.org/10.1016/j.trd.2020.102221

Hetz, Patricia R.; Dawson, Christi L.; Cullen, Theresa A. (2015). "Social media use and the fear of missing out (FoMO) while studying abroad". Journal of research on technology in education, v. 47, n. 4, pp. 259-272. https://doi.org/10.1080/15391523.2015.1080585

Hiremath, Pavan; Kowshik, C. S. Suhas; Manjunath, Maitri (2020). “Covid 19: Impact of lock-down on mental health and tips to overcome". Asian journal of psychiatry, v. 51, 102088.

https://doi.org/10.1016/j.ajp.2020.102088 
Hu, Minqing; Liu, Bing (2004). "Mining and summarizing customer reviews". In: Proceedings of the $10^{\text {th }}$ ACM Sigkdd International conference on knowledge discovery and data mining, pp. 168-177.

https://doi.org/10.1145/1014052.1014073

Jin, Songchang; Lin, Wangqun; Yin, Hong; Yang, Shuqiang; Li, Aiping; Deng, Bo (2015). “Community structure mining in big data social media networks with MapReduce". Cluster computing, v. 18, n. 3, pp. 999-1010.

https://doi.org/10.1007/s10586-015-0452-x

Katz, Vikki S.; González, Carmen (2016). "Toward meaningful connectivity: Using multilevel communication research to reframe digital inequality". Journal of communication, v. 66, n. 2, pp. 236-249.

https://doi.org/10.1111/jcom.12214

Keegan, Brendan J.; Rowley, Jennifer (2017). "Evaluation and decision making in social media marketing". Management decision, v. 55, n. 1, pp. 15-31.

https://doi.org/10.1108/MD-10-2015-0450

Kim, Jooho; Hastak, Makarand (2018). "Social network analysis: Characteristics of online social networks after a disaster". International journal of information management, v. 38, n. 1, pp. 86-96.

https://doi.org/10.1016/j.ijinfomgt.2017.08.003

King, Anna-Lucia-Spear; Valenca, Alexandre-Martins; Silva, Adriana-Cardoso O.; Baczynski, Tatiana-Pires; De-Carvalho, Marcele-Regine; Nardi, Antonio E. (2013). “Nomophobia: Dependency on virtual environments or social phobia?”. Computers in human behavior, v. 29, n. 1, pp. 140-144.

https://doi.org/10.1016/j.chb.2012.07.025

Kireyev, Kirill; Palen, Leysia; Anderson, Kenneth M. (2009). "Applications of topics models to analysis of disaster-related Twitter data". Paper presented at the NIPS Workshop on applications for topic models: text and beyond.

Latorre-Martínez, Pilar; Orive-Serrano, Víctor; Íñiguez-Dieste, David (2018). “Medición y análisis de la audiencia social de las televisiones autonómicas en Facebook y Twitter". El profesional de la información, v. 27, n. 5, pp. $1061-1070$.

https://doi.org/10.3145/epi.2018.sep.10

Liu, Wenlin; Lai, Chih-Hui; Xu, Weiai (2018). "Tweeting about emergency: A semantic network analysis of government organizations' social media messaging during hurricane Harvey". Public relations review, v. 44, n. 5, pp. 807-819.

https://doi.org/10.1016/j.pubrev.2018.10.009

López-Pérez, Lourdes; Olvera-Lobo, María-Dolores (2016). "Social media as channels for the public communication of science. The case of Spanish research centers and public universities". In: Knautz, Kathrin; Baran, Katsiaryna S. (eds.). Facets of Facebook: use and users, pp. 241-264.

http://hdl.handle.net/10481/43871

Mehra, Aseem; Rani, Seema; Sahoo, Swapnajeet; Parveen, Shaheena; Singh, Ajay-Pal; Chakrabarti, Subho; Grover, Sandeep (2020). "A crisis for elderly with mental disorders: Relapse of symptoms due to heightened anxiety due to COVID-19". Asian journal of psychiatry, v. 51, 102114.

https://doi.org/10.1016/j.ajp.2020.102114

Moghanibashi-Mansourieh, Amir (2020). "Assessing the anxiety level of Iranian general population during Covid-19 outbreak". Asian journal of psychiatry, v. 51, 102076.

https://doi.org/10.1016/j.ajp.2020.102076

NHPCO (2020). Coronavirus disease 2019 (Covid-19) Shared decision-making tool. National Hospice and Palliative Care Organization.

https://www.nhpco.org/wpcontent/uploads/COVID-19-Shared-Decision-Making-Tool.pdf

Oradini, Federica; Saunders, Gunter (2008). "The use of social networking by students and staff in higher education". Paper presented at the iLearning forum.

Pourebrahim, Nastaran; Sultana, Selima; Edwards, John; Gochanour, Amanda; Mohanty, Somya (2019). "Understanding communication dynamics on Twitter during natural disasters: A case study of hurricane Sandy". International journal of disaster risk reduction, v. 37, 101176.

https://doi.org/10.1016/j.ijdrr.2019.101176

Qiu, Jianyin; Shen, Bin; Zhao, Min; Wang, Zhen; Xie, Bin; Xu, Yifeng (2020). “A nationwide survey of psychological distress among Chinese people in the Covid-19 epidemic: Implications and policy recommendations". General psychiatry, v. 33 , n. 2. https://doi.org/10.1136/gpsych-2020-100213

Reynard, Darcy; Shirgaokar, Manish (2019). "Harnessing the power of machine learning: Can Twitter data be useful in guiding resource allocation decisions during a natural disaster?". Transportation research part D: Transport and environment, v. 77, pp. 449-463.

https://doi.org/10.1016/j.trd.2019.03.002 
Rodríguez-Vázquez, Clide; Teijeiro-Álvarez, Mercedes; Blázquez-Lozano, Félix (2018). “Evaluation of the fanpages of Spanish universities: Public versus private institutions". Universal access in the information society, v. 17, n. 4, pp. 735-743. https://doi.org/10.1007/s10209-017-0530-3

Roy, Deblina; Tripathy, Sarvodaya; Kar, Sujita-Kumar; Sharma, Nivedita; Verma, Sudhir-Kumar; Kaushal, Vikas (2020). "Study of knowledge, attitude, anxiety y perceived mental healthcare need in Indian population during Covid-19 pandemic". Asian journal of psychiatry, v. 51, 102083.

https://doi.org/10.1016/j.ajp.2020.102083

Ruz, Gonzalo A.; Henríquez, Pablo A.; Mascareño, Aldo (2020). "Sentiment analysis of Twitter data during critical events through Bayesian networks classifiers". Future generation computer systems, v. 106, pp. 92-104.

https://doi.org/10.1016/j.future.2020.01.005

Saxton, Gregory D.; Guo, Chao (2014). "Online stakeholder targeting and the acquisition of social media capital". International journal of nonprofit and voluntary sector marketing, v. 19, n. 4, pp. 286-300.

https://doi.org/10.1002/nvsm.1504

Sedereviciute, Kristina; Valentini, Chiara (2011). "Towards a more holistic stakeholder analysis approach. Mapping known and undiscovered stakeholders from social media". International journal of strategic communication, v. 5, n. 4, pp. 221-239.

https://doi.org/10.1080/1553118X.2011.592170

Singh, Neha; Roy, Nirmalya; Gangopadhyay, Aryya (2019). "Analyzing the emotions of crowd for improving the emergency response services". Pervasive and mobile computing, v. 58, 101018.

https://doi.org/10.1016/j.pmcj.2019.04.009

Sola-Morales, Salomé; Rivera-Gallardo, Ricardo (2015). "Las redes sociales como catalizador del movimiento estudiantil chileno en 2011". Chasqui: revista latinoamericana de comunicación, v. 128, pp. 37-52.

https://revistachasqui.org/index.php/chasqui/article/view/2243

Takahashi, Bruno; Tandoc, Edson C.; Carmichael, Christine (2015). "Communicating on Twitter during a disaster: An analysis of tweets during typhoon Haiyan in the Philippines". Computers in human behavior, v. 50, pp. 392-398. https://doi.org/10.1016/j.chb.2015.04.020

Theja-Bhavaraju, Sai-Krishna; Beyney, Cyril; Nicholson, Charles (2019). "Quantitative analysis of social media sensitivity to natural disasters". International journal of disaster risk reduction, v. 39, 101251.

https://doi.org/10.1016/j.ijdrr.2019.101251

Thelwall, Mike; Levitt, Jonathan M. (2020). "Retweeting Covid-19 disability issues: Risks, support and outrage". El profesional de la información, v. 29, n. 2, e290216.

https://doi.org/10.3145/epi.2020.mar.16

Trainor, Kevin J.; Andzulis, James M.; Rapp, Adam; Agnihotri, Raj (2014). "Social media technology usage and customer relationship performance: A capabilities-based examination of social CRM". Journal of business research, v. 67, n. 6, pp. 1201-1208.

https://doi.org/10.1016/j.jbusres.2013.05.002

Vijaykumar, Santosh; Nowak, Glen; Himelboim, Itai; Jin, Yan (2018). "Virtual Zika transmission after the first U.S. case: Who said what and how it spread on Twitter". American journal of infection control, v. 46, n. 5, pp. 549-557. https://doi.org/10.1016/j.ajic.2017.10.015

Wallace, Cara L.; Waldkowski, Stephanie P.; Gibson, Allison; White, Patrick (2020). "Grief during the Covid-19 pandemic: Considerations for palliative care providers". Journal of pain and symptom management, v. 60, n. 1, E70-E76. https://doi.org/10.1016/j.jpainsymman.2020.04.012

Wamba, Samuel-Fosso; Akter, Shahriar; Edwards, Andrew; Chopin, Geoffrey; Gnanzou, Denis (2015). "How 'big data' can make big impact: Findings from a systematic review and a longitudinal case study". International journal of production economics, v. 165, pp. 234-246.

https://doi.org/10.1016/j.ijpe.2014.12.031

Wang, C. Jason; Ng, Chun Y.; Brook, Robert H. (2020). "Response to Covid-19 in Taiwan: Big data analytics, new technology, and proactive testing". JAMA. Journal of the American Medical Association, v. 323, n. 14, pp. 1341-1342. https://doi.org/10.1001/jama.2020.3151

Wang, Zhicheng; Tang, Kun (2020). “Combating Covid-19: Health equity matters". Nature medicine, v. 26, n. 4, 458. https://doi.org/10.1038/s41591-020-0823-6

Weaver, Meaghann S.; Wiener, Lori (2020). "Applying palliative care principles to communicate with children about Covid-19". Journal of pain and symptom management, v. 60, n. 1.

https://doi.org/10.1016/j.jpainsymman.2020.03.020 
Welton-Mitchell, Courtney; James, Leah-Emily; Khanal, Shree-Niwas; James, Alexander-Scott (2018). “An integrated approach to mental health and disaster preparedness: A cluster comparison with earthquake affected communities in Nepal". BMC psychiatry, v. 18, n. 1, pp. 1-14.

https://doi.org/10.1186/s12888-018-1863-z

White, David S.; Le-Cornu, Alison (2011). "Visitors and residents: A new typology for online engagement". First Monday, v. 9, n. 16. https://firstmonday.org/article/view/3171/3049

Wu, Desheng; Cui, Yiwen (2018). "Disaster early warning and damage assessment analysis using social media data and geo-location information". Decision support systems, v. 111, pp. 48-59.

https://doi.org/10.1016/j.dss.2018.04.005

Yu, Yang; Duan, Wenjing; Cao, Qing (2013). "The impact of social and conventional media on firm equity value: A sentiment analysis approach". Decision support systems, v. 55, n. 4, pp. 919-926.

https://doi.org/10.1016/j.dss.2012.12.028

\section{Anexo 1. Cuentas corporativas monitorizadas}

\begin{tabular}{|c|c|c|c|}
\hline Comunidad autónoma & Universidad & Titularidad & Perfil en Twitter \\
\hline \multirow{10}{*}{ Andalucía } & Universidad de Almería & Pública & @ualmeria \\
\hline & Universidad de Cádiz & Pública & @univcadiz \\
\hline & Universidad de Córdoba & Pública & @Univcordoba \\
\hline & Universidad de Granada & Pública & @CanalUGR \\
\hline & Universidad de Huelva & Pública & @UniHuelva \\
\hline & Universidad de Jaén & Pública & @ujaen \\
\hline & Universidad de Málaga & Pública & @InfoUMA \\
\hline & Universidad de Sevilla & Pública & @unisevilla \\
\hline & Universidad Internacional de Andalucía & Privada & @UNIAuniversidad \\
\hline & Universidad Pablo de Olavide & Privada & @pablodeolavide \\
\hline \multirow{2}{*}{ Aragón } & Universidad de Zaragoza & Pública & @unizar \\
\hline & Universidad San Jorge & Privada & @_usj_ \\
\hline Cantabria & Universidad de Cantabria & Pública & @unican \\
\hline Castilla la Mancha & Universidad de Castilla-La Mancha & Pública & @uclm_es \\
\hline \multirow{8}{*}{ Castilla y León } & IE Universidad & Privada & @IEuniversity \\
\hline & Universidad Católica de Ávila & Privada & @UCAVILA \\
\hline & Universidad de Burgos & Pública & @UBUEstudiantes \\
\hline & Universidad de León & Pública & @unileon \\
\hline & Universidad de Salamanca & Pública & @usal \\
\hline & Universidad de Valladolid & Pública & @UVa_es \\
\hline & Universidad Europea Miguel de Cervantes & Privada & @UEMC \\
\hline & Universidad Pontificia de Salamanca & Privada & @upsa \\
\hline \multirow{11}{*}{ Cataluña } & Universitat Autònoma de Barcelona & Pública & @UABBarcelona \\
\hline & Universitat de Barcelona & Pública & @UniBarcelona \\
\hline & Universitat de Girona & Pública & @univgirona \\
\hline & Universitat de Vic & Privada & @uvic_ucc \\
\hline & Universitat Internacional de Catalunya & Privada & @UICbarcelona \\
\hline & Universitat Pompeu Fabra & Pública & @UPFBarcelona \\
\hline & Universitat Ramon Llull & Privada & @uramonllull \\
\hline & Universitat Rovira i Virgili & Pública & @universitatURV \\
\hline & Universitat Abat Oliba CEU & Privada & @UAOCEU \\
\hline & Universitat de Lleida & Pública & @UdL_info \\
\hline & Universitat Oberta de Catalunya & Privada & @UOCuniversitat \\
\hline \multirow{2}{*}{$\begin{array}{l}\text { Comunidad Autónoma de } \\
\text { Canarias }\end{array}$} & Universidad de La Laguna & Pública & @CanalULL \\
\hline & Universidad de Las Palmas de Gran Canaria & Pública & @ULPGC \\
\hline
\end{tabular}




\begin{tabular}{|c|c|c|c|}
\hline \multirow{16}{*}{ Comunidad de Madrid } & Universidad a Distancia de Madrid & Privada & @UDIMA \\
\hline & Universidad Alfonso XEI Sabio & Privada & @universidad_uax \\
\hline & Universidad Antonio de Nebrija & Privada & $@$ @ebrija \\
\hline & Universidad Autónoma de Madrid & Pública & @UAM_Madrid \\
\hline & Universidad Camilo José Cela & Privada & @universidadcjc \\
\hline & Universidad Carlos III de Madrid & Pública & @uc3m \\
\hline & Universidad Complutense de Madrid & Pública & @unicomplutense \\
\hline & Universidad de Alcalá & Pública & @UAHes \\
\hline & Universidad Europea de Madrid & Privada & @UEuropea \\
\hline & Universidad Francisco de Vitoria & Privada & @ufvmadrid \\
\hline & Universidad Internacional Menéndez Pelayo & Pública & $@$ @UIMP \\
\hline & Universidad Nacional de Educación a Distancia & Pública & $@$ @UNED \\
\hline & Universidad Politécnica de Madrid & Pública & @La_UPM \\
\hline & Universidad Pontificia Comillas & Privada & @UCOMILLAS \\
\hline & Universidad Rey Juan Carlos & Pública & @urjc \\
\hline & Universidad San Pablo CEU & Privada & @USPCEU \\
\hline \multirow{2}{*}{ Comunidad Foral de Navarra } & Universidad de Navarra & Privada & @unav \\
\hline & Universidad Pública de Navarra & Pública & @Unavarra \\
\hline \multirow{7}{*}{ Comunidad Valenciana } & Universidad Cardenal Herrera - CEU & Privada & @uchceu \\
\hline & Universidad Católica de Valencia San Vicente Mártir & Privada & @UCV_es \\
\hline & Universidad de Alicante & Pública & @UA_Universidad \\
\hline & Universidad Miguel Hernández de Elche & Pública & @UniversidadMH \\
\hline & Universidad Politécnica de Valéncia & Pública & @UPV \\
\hline & Universitat de València & Pública & @UV_EG \\
\hline & Universitat Jaume I de Castelló & Pública & @UJI_noticies \\
\hline Extremadura & Universidad de Extremadura & Pública & @Infouex \\
\hline \multirow{3}{*}{ Galicia } & Universidade da Coruña & Pública & @UDC_gal \\
\hline & Universidade de Santiago de Compostela & Pública & @UniversidadeUSC \\
\hline & Universidade de Vigo & Pública & @uvigo \\
\hline Islas Baleares & Universitat de les Illes Balears & Pública & @UIBuniversitat \\
\hline La Rioja & Universidad de La Rioja & Pública & @unirioja \\
\hline \multirow{3}{*}{ País Vasco } & Universidad de Deusto & Privada & @deusto \\
\hline & Universidad Mondragon Unibertsitatea & Privada & $@ M U n i b e r t s i t a t e a$ \\
\hline & Universidad del País Vasco & Pública & @upvehu \\
\hline Principado de Asturias & Universidad de Oviedo & Pública & @uniovi_info \\
\hline \multirow{3}{*}{ Región de Murcia } & Universidad Católica San Antonio de Murcia & Privada & @UCAM \\
\hline & Universidad de Murcia & Pública & $@ U M U$ \\
\hline & Universidad Politécnica de Cartagena & Pública & @UPCTnoticias \\
\hline
\end{tabular}


Anexo 2. Media de los indicadores clave del rendimiento en Twitter de las universidades españolas durante los meses de enero, febrero, marzo y abril de 2020

\begin{tabular}{|c|c|c|c|c|c|c|}
\hline Universidades & Perfil en Twitter & $\begin{array}{c}\text { (KPI 1) } \\
\text { Compro- } \\
\text { miso }^{*}\end{array}$ & $\begin{array}{c}\text { (KPI 2) } \\
\text { Número } \\
\text { de segui- } \\
\text { dores }\end{array}$ & $\begin{array}{c}\text { (KPI 3) } \\
\text { Total } \\
\text { tweets }\end{array}$ & $\begin{array}{l}\text { (KPI 4) } \\
\text { Total "fa- } \\
\text { voritos" }\end{array}$ & $\begin{array}{c}\text { (KPI 5) } \\
\text { Total } \\
\text { retweets }\end{array}$ \\
\hline \multicolumn{7}{|l|}{ Andalucía } \\
\hline Universidad de Almería & Universidad de Almería & $0,06 \%$ & 36.347 & 282 & 1.645 & 803 \\
\hline Universidad de Cádiz & Universidad de Cádiz & $0,07 \%$ & 50.482 & 463 & 2.881 & 1.191 \\
\hline Universidad de Córdoba & Universidad Córdoba & $0,14 \%$ & 48.687 & 972 & 5.088 & 3.006 \\
\hline Universidad de Granada & Universidad Granada & $0,10 \%$ & 132.029 & 593 & 9.830 & 5.223 \\
\hline Universidad de Huelva & Univ. de Huelva UHU & $0,07 \%$ & 33.598 & 665 & 1.724 & 1.124 \\
\hline Universidad de Jaén & Universidad de Jaén & $0,10 \%$ & 50.151 & 616 & 4.199 & 2.073 \\
\hline Universidad de Málaga & Universidad Málaga & $0,10 \%$ & 119.898 & 587 & 9.499 & 5.468 \\
\hline Universidad de Sevilla & unisevilla & $0,13 \%$ & 127.512 & 4.272 & 12.969 & 7.357 \\
\hline Universidad Internacional de Andalucía & UNIA & $0,13 \%$ & 10.591 & 371 & 1.095 & 572 \\
\hline Universidad Pablo de Olavide & Pablo de Olavide & $0,16 \%$ & 30.611 & 1.038 & 3.981 & 1.904 \\
\hline \multicolumn{7}{|l|}{ Aragón } \\
\hline Universidad de Zaragoza & Universidad Zaragoza & $0,43 \%$ & 23.106 & 771 & 8.049 & 3.472 \\
\hline Universidad San Jorge & UniversidadSanJorge & $0,38 \%$ & 3.959 & 304 & 1.169 & 594 \\
\hline \multicolumn{7}{|l|}{ Asturias } \\
\hline Universidad de Oviedo & Universidad Oviedo & $0,41 \%$ & 13.835 & 845 & 4.300 & 2.226 \\
\hline \multicolumn{7}{|l|}{ Cantabria } \\
\hline Universidad de Cantabria & unican.es & $0,31 \%$ & 14.090 & 453 & 3.595 & 1.498 \\
\hline \multicolumn{7}{|l|}{ Castilla-La Mancha } \\
\hline Universidad de Castilla-La Mancha & Universidad de Castilla-La Mancha & $0,14 \%$ & 46.134 & 440 & 5.340 & 2.628 \\
\hline \multicolumn{7}{|l|}{ Castilla y León } \\
\hline IE University & IEuniversity & $0,09 \%$ & 13.287 & 121 & 920 & 421 \\
\hline Universidad de Valladolid & $\begin{array}{l}\text { Gabinete de Comunicación Univer- } \\
\text { sidad de Valladolid }\end{array}$ & $0,09 \%$ & 48.715 & 246 & 2.752 & 2.342 \\
\hline Universidad Católica de Ávila & Universidad de Ávila & $0,14 \%$ & 10.730 & 830 & 1.341 & 453 \\
\hline Universidad Europea Miguel de Cervantes & UEMC & $0,31 \%$ & 7.290 & 594 & 1.864 & 812 \\
\hline Universidad de Burgos & Universidad Burgos & $0,21 \%$ & 12.369 & 2.502 & 2.028 & 1.113 \\
\hline Universidad de León & Universidad de León & $0,11 \%$ & 31.447 & 855 & 2.725 & 1.414 \\
\hline Universidad de Salamanca & Universidad de Salamanca & $0,24 \%$ & 57.660 & 754 & 10.480 & 6.216 \\
\hline Universidad Pontificia de Salamanca & UPSA & $0,28 \%$ & 5.396 & 644 & 1.215 & 535 \\
\hline \multicolumn{7}{|l|}{ Cataluña } \\
\hline UIC Barcelona & UICbarcelona & $0,04 \%$ & 15.800 & 201 & 625 & 227 \\
\hline Universitat Abat Oliba CEU & UAO CEU & $0,04 \%$ & 5.007 & 119 & 206 & 54 \\
\hline Universitat Autònoma de Barcelona & Universitat Autònoma de Barcelona & $0,25 \%$ & 49.478 & 1.721 & 10.534 & 3.776 \\
\hline Universitat de Barcelona & Universitat de Barcelona & $0,19 \%$ & 59.030 & 774 & 9.189 & 4.290 \\
\hline Universitat de Girona & Universitat de Girona & $0,13 \%$ & 40.912 & 1.066 & 4.043 & 2.093 \\
\hline Universitat de Lleida & UdL Informació & $0,40 \%$ & 4.903 & 379 & 1.451 & 849 \\
\hline Universitat Oberta de Catalunya & UOCuniversitat & $0,17 \%$ & 36.212 & 908 & 5.255 & 2.307 \\
\hline Universitat Pompeu Fabra & UPF Barcelona & $0,46 \%$ & 36.721 & 924 & 14.285 & 5.643 \\
\hline Universitat Ramon Llull & U. Ramon Llull (URL) & $0,16 \%$ & 11.300 & 1.412 & 1.423 & 730 \\
\hline Universitat Rovira i Virgili & Universitat URV & $0,30 \%$ & 16.571 & 660 & 3.929 & 2.025 \\
\hline \multicolumn{7}{|l|}{ Extremadura } \\
\hline Universidad de Extremadura & UEx & $0,21 \%$ & 20.272 & 536 & 2.932 & 2.030 \\
\hline \multicolumn{7}{|l|}{ Galicia } \\
\hline Universidade da Coruña & Universidade da Coruña & $0,21 \%$ & 33.731 & 660 & 5.470 & 3.054 \\
\hline Universidade de Santiago de Compostela & USC & $0,34 \%$ & 15.428 & 192 & 3.944 & 2.037 \\
\hline Universidade de Vigo & Universidade de Vigo & $0,11 \%$ & 39.265 & 774 & 2.925 & 2.211 \\
\hline
\end{tabular}




\begin{tabular}{|c|c|c|c|c|c|c|}
\hline \multicolumn{7}{|l|}{ La Rioja } \\
\hline Universidad de La Rioja & Universidad de La Rioja & $0,10 \%$ & 25.568 & 1.071 & 2.139 & 940 \\
\hline \multicolumn{7}{|l|}{ Madrid } \\
\hline Nebrija Universidad & Universidad Nebrija & $0,2 \%$ & 16.875 & 963 & 2.977 & 1.022 \\
\hline UMH Universidad Miguel Hernández & UMH & $0,17 \%$ & 26.364 & 660 & 3.807 & 1.581 \\
\hline $\begin{array}{l}\text { Universidad Nacional de Educación a } \\
\text { Distancia }\end{array}$ & UNED & $0,13 \%$ & 110.708 & 1.066 & 11.073 & 5.684 \\
\hline Universidad Alfonso X el Sabio & UAX & $0,24 \%$ & 6.784 & 164 & 1.318 & 578 \\
\hline Universidad Autónoma de Madrid & UAM Autónoma Madrid & $0,12 \%$ & 56.744 & 417 & 5.542 & 2.596 \\
\hline Universidad Camilo José Cela & UCJC & $0,10 \%$ & 17.127 & 362 & 1.573 & 567 \\
\hline Universidad Carlos III de Madrid & UC3M & $0,06 \%$ & 38.543 & 257 & 1.836 & 816 \\
\hline Universidad San Pablo CEU & Universidad USPCEU & $0,14 \%$ & 11.659 & 175 & 1.314 & 615 \\
\hline Universidad Complutense de Madrid & Complutense & $0,18 \%$ & 89.731 & 325 & 13.865 & 5.761 \\
\hline Universidad de Alcalá & Universidad Alcalá & $0,1 \%$ & 54.958 & 538 & 4.415 & 2.035 \\
\hline Universidad Europea de Madrid & Universidad Europea & $0,06 \%$ & 43.420 & 861 & 2.336 & 835 \\
\hline Universidad Francisco de Vitoria & Francisco de Vitoria & $0,23 \%$ & 11.446 & 649 & 2.232 & 890 \\
\hline $\begin{array}{l}\text { Universidad Internacional Menéndez } \\
\text { Pelayo }\end{array}$ & UIMP & $0,00 \%$ & 22.888 & 98 & 30 & 12 \\
\hline Universidad Politécnica de Madrid & Politécnica Madrid & $0,31 \%$ & 42.263 & 1.057 & 9.667 & 6.127 \\
\hline Universidad Pontificia Comillas & Universidad Pontificia Comillas & $0,33 \%$ & 10.957 & 756 & 2.926 & 1.287 \\
\hline Universidad Rey Juan Carlos & URJC & $0,09 \%$ & 51.481 & 401 & 3.701 & 1.673 \\
\hline Universidad a Distancia de Madrid & Universidad UDIMA & $0,17 \%$ & 14.835 & 585 & 1.980 & 1.029 \\
\hline \multicolumn{7}{|l|}{ Murcia } \\
\hline $\begin{array}{l}\text { Universidad Católica San Antonio de } \\
\text { Murcia }\end{array}$ & UCAM Universidad & $0,28 \%$ & 34.889 & 1.302 & 7.990 & 3.794 \\
\hline Universidad de Murcia & Universidad Murcia & $0,09 \%$ & 104.996 & 2.100 & 7.300 & 4.117 \\
\hline Universidad Politécnica de Cartagena & $\begin{array}{l}\text { UPCT | Universidad Politécnica de } \\
\text { Cartagena }\end{array}$ & $0,56 \%$ & 7.553 & 639 & 2.957 & 2.047 \\
\hline \multicolumn{7}{|l|}{ Navarra } \\
\hline Universidad de Navarra & Universidad de Navarra & $0,15 \%$ & 46.945 & 327 & 6.171 & 2.257 \\
\hline Universidad Pública de Navarra & UPNA - Universidad & $0,10 \%$ & 15.722 & 483 & 1.344 & 485 \\
\hline \multicolumn{7}{|l|}{ País Vasco } \\
\hline Universidad Mondragon Unibertsitatea & Mondragon Unibertsitatea & $0,14 \%$ & 9.150 & 385 & 962 & 601 \\
\hline Universidad de Deusto & Universidad Deusto & $0,19 \%$ & 26.618 & 458 & 4.143 & 2.004 \\
\hline Universidad del País Vasco & UPV/EHU & $0,19 \%$ & 28.047 & 253 & 3.953 & 2.211 \\
\hline \multicolumn{7}{|l|}{ Valencia } \\
\hline Universitat d'Alacant & Universidad de Alicante UA & $0,11 \%$ & 1056 & 301 & 9.530 & 3.842 \\
\hline Universidad CEU Cardenal Herrera & Universidad CEU UCH & $0,08 \%$ & 13.322 & 704 & 842 & 358 \\
\hline Universitat de València & Universitat València & $0,06 \%$ & 66.017 & 357 & 3.145 & 1.702 \\
\hline Universitat Jaume I & Universitat Jaume I & $0,41 \%$ & 16.419 & 2.475 & 5.406 & 2.650 \\
\hline $\begin{array}{l}\text { Universidad Católica de Valencia San } \\
\text { Vicente Mártir }\end{array}$ & Universidad Católica de Valencia & $0,15 \%$ & 11.408 & 986 & 1.522 & 581 \\
\hline Universitat Politècnica de València & Universitat Politècnica de València & $0,25 \%$ & 49.818 & 1.054 & 10.351 & 4.654 \\
\hline \multicolumn{7}{|l|}{ Islas Baleares } \\
\hline Universitat de les Illes Balears & Universitat de les Illes Balears - UIB & $0,40 \%$ & 17.527 & 471 & 5.417 & 2.890 \\
\hline \multicolumn{7}{|l|}{ Islas Canarias } \\
\hline ULPGC & ULPGC & $0,12 \%$ & 50.737 & 1.411 & 5.006 & 2.253 \\
\hline Universidad de La Laguna & Universidad de La Laguna & $0,14 \%$ & 49.408 & 1.471 & 5.220 & 2.748 \\
\hline Media & & $0,186 \%$ & 36.313 & 766 & 4.429 & 2.181 \\
\hline Desviación típica & & $0,117 \%$ & 30.344 & 647 & 3.479 & 1.727 \\
\hline
\end{tabular}

* El compromiso muestra un promedio de la frecuencia con que un seguidor interactúa con la cuenta. Se calcula dividiendo la cantidad diaria de retweets y favoritos por el número de seguidores. 


\section{Anexo 3. Top 15 posts según número de "me gusta" en el período analizado}

\begin{tabular}{|c|c|c|c|c|}
\hline Fecha & Tweet & Cuenta & Favoritos & Covid-19 \\
\hline $27 \mathrm{feb}$ & $\begin{array}{l}\text { Davant les informacions aparegudes avui, } 27 \text { de feb., en relació a les activitats del prof. } \\
\text { Andrés Betancor, el rector de la UPF ha ordenat l'obertura d'un expedient informatiu per } \\
\text { aclarir si les actuacions d'aquest professor poden ser objecte de sanció per part de la } \\
\text { Universitat }\end{array}$ & 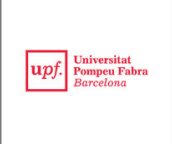 & 3.697 & \\
\hline $12 \mathrm{mar}$ & $\begin{array}{l}\text { \#UniBarcelona |?? D’acord amb la informació del @govern de la Generalitat us avancem } \\
\text { que la UB suspendrà l'activitat acadèmica presencial a partir de demà divendres } 13 \text { de } \\
\text { març i durant dues setmanes. }\end{array}$ & $\begin{array}{c}\text { 需 } \\
\text { UNIVRELITAT. } \\
\text { BARCELONA }\end{array}$ & 1.192 & $x$ \\
\hline $27 \mathrm{feb}$ & $\begin{array}{l}\text { El rector Jaume Casals ha expressat la seva profunda preocupació personal davant la } \\
\text { possibilitat que un profesor de la comunitat pugui tenir una actuació èticament tan } \\
\text { reprobable. }\end{array}$ & $\begin{array}{l}\text { niversitat } \\
\text { omperutarabra } \\
\text { arrelona }\end{array}$ & 1.109 & \\
\hline $13 \mathrm{abr}$ & $\begin{array}{l}\text { @antoniobanderas y @pabloalboran colaborarán con } 200.000 \text { euros en las investiga- } \\
\text { ciones del equipo del Grupo de Robótica Médica de la UMA, liderado por Víctor Muñoz, } \\
\text { que ha hecho posible el respirador 'Andalucía Respira'. +info: https://t.co/LeVCnECVmT } \\
\text { https://t.co/sPo3FTAVue }\end{array}$ & $\begin{array}{l}\text { NVERSIDAC } \\
\text { EMALAGA }\end{array}$ & 867 & $x$ \\
\hline $12 \mathrm{mar}$ & $\begin{array}{l}\text { Seguint les instruccions del @govern, la \#UAB suspèn l'activitat lectiva presencial de } \\
\text { manera indefinida des del } 13 \text { de març de } 2020 \text { (inclòs) a tots els centres. La docència } \\
\text { s'impartirà de forma virtual. (1/4) https://t.co/zaAMdBxND6 }\end{array}$ & $\begin{array}{l}\text { UAB } \\
\text { Universitat } \\
\text { Autònoma } \\
\text { de Barcelona }\end{array}$ & 765 & $x$ \\
\hline $22 \mathrm{mar}$ & $\begin{array}{l}\text { La \#UAB, @CRAGENOMICA i @_IFAE envien material de protecció al personal sanitari } \\
\text { de @parctauli, @hgermanstrias, @hospitaldelmar, @HospitalSantPau i @vallhebron. } \\
\text { Endavant! ?? } \\
\text { \#orgullUAB \#joemquedoacasa \#quedatacasa https://t.co/j5FqZR4rf5 }\end{array}$ & $\begin{array}{l}\text { UAB } \\
\text { Universitat } \\
\text { Autônoma } \\
\text { de Barcelona }\end{array}$ & 759 & $x$ \\
\hline 06 mar & $\begin{array}{l}\text { \#DavisHonorisUPF } \\
\text { Angela Y. Davis, activista política nord-americana, serà investida doctora honoris causa } \\
\text { per la \#UPFBarcelona el } 12 \text { de maig a l'auditori del campus de la Ciutadella. } \\
\text { https://t.co/HfQ50iph8B https://t.co//1gLGMtCGR }\end{array}$ & 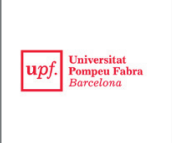 & 720 & \\
\hline $12 \mathrm{mar}$ & $\begin{array}{l}\text { \#COMUNICADO | El Rector de la Universidad de Salamanca, Ricardo Rivero, comunica } \\
\text { la suspensión provisional de todas las actividades lectivas hasta que las autoridades } \\
\text { sanitarias se pronuncien. https://t.co/xXjUJy5JTg }\end{array}$ & 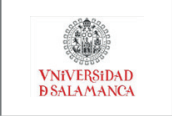 & 678 & $x$ \\
\hline 27 ene & $\begin{array}{l}\text { Hatsune Miku @cfm_miku_en will perform live tomorrow in \#Barcelona. Her voice was } \\
\text { created using @vocaloid, a technology developed by \#Yamaha Corporation y the @ } \\
\text { mtg_upf at \#UPFBarcelona. } \\
\text { https://t.co/R47GCDJd1b \#MIKUEXPO2020 https://t.co/QqMgMMoB5d }\end{array}$ & upp. $\begin{array}{l}\text { Universitat } \\
\text { Pompout arabra } \\
\text { Barcelona }\end{array}$ & 662 & \\
\hline 30 mar & $\begin{array}{l}\text { El respirador automático 'Málaga Respira', desarrollado en menos de una semana por } \\
\text { investigadores UMA, científicos de @_ibima y profesionales sanitarios, pasa con éxito } \\
\text { el primer ensayo clínico con un paciente real en el Hospital de Antequera https://t.co/ } \\
\text { JDwGdRUikf } \\
\text { \#COVID-19 https://t.co/azjOwOzlqe }\end{array}$ & O. UNIVERIDAD & 515 & $x$ \\
\hline $10 \mathrm{abr}$ & $\begin{array}{l}\text { El respirador artificial desarrollado por investigadores UMA junto a @_ibima y sanita- } \\
\text { rios ya cuenta con la autorización de la Agencia del Medicamento (@AEMPSGOB), tras } \\
\text { superar con éxito todos los requerimientos necesarios para su homologación: https://t. } \\
\text { co/rJAflrYac0 } \\
\text { \#COVID-19 https://t.co/wMAx5qtgRx }\end{array}$ & $\begin{array}{l}\text { UNVERSIIAD } \\
\text { DE WALAGA }\end{array}$ & 508 & $x$ \\
\hline $07 \mathrm{abr}$ & $\begin{array}{l}\text { Un equipo de científicos de la Universidad Autónoma de Madrid ha hallado un com- } \\
\text { puesto que impide la entrada de los virus en las células. El polímero natural, que está en } \\
\text { proceso de ser patentado, podría abrir nuevas vías para encontrar un antiviral frente al } \\
\text { coronavirus. https://t.co/dQZXDLITGt }\end{array}$ & $\underset{\substack{\text { Universidad Autónoma } \\
\text { de Madrid }}}{\mathbf{U} A \mathbf{M}}$ & 490 & $x$ \\
\hline 22 ene & $\begin{array}{l}\text { La comunitat de la \#UPFBarcelona lamenta la sobtada mort de David Caminada, @ } \\
\text { alumniupf i professor associat de @ComunicacioUPF. Compartim el sentiment per la } \\
\text { seva pèrdua i volem expressar el nostre condol a família i amics. \#DEP @bcn_ajuntament } \\
\text { https://t.co/i47Herd1BI }\end{array}$ & upf. $\begin{array}{c}\text { Universitat } \\
\text { Ponper a abra } \\
\text { Barcelona }\end{array}$ & 485 & \\
\hline $06 \mathrm{feb}$ & $\begin{array}{l}\text { La tesis de nuestra doctora María del Mar Haro Soler, ha sido finalmente reconocida } \\
\text { como la mejor tesis del mundo en Traducción e Interpretación. Recibirá el galardón tras } \\
\text { la presentación de su trabajo en la CIUTI Conference en ISIT Paris (2-5 junio). ¡Enhora- } \\
\text { buena! https://t.co/oUSS1TQtm7 }\end{array}$ & Universidad de Granada & 483 & \\
\hline 12 mar & $\begin{array}{l}\text { La UIB suspèn l'activitat acadèmica presencial des de dilluns, } 16 \text { de març de 2020, durant } \\
\text { dues setmanes. La desició s'ha pres arran de la compareixença de la Presidenta @F_Ar- } \\
\text { mengol. Ampliarem informació a la web @UIBuniversitat i canals oficials. }\end{array}$ & 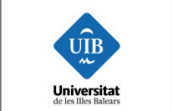 & 477 & $x$ \\
\hline
\end{tabular}

